\title{
On the scaling of the instability of a flat sediment bed with respect to ripple-like patterns
}

\author{
Markus Scherer $^{1}$, Aman G. Kidanemariam ${ }^{2}$ and Markus Uhlmann ${ }^{1}{ }^{\dagger}$ \\ ${ }^{1}$ Institute for Hydromechanics, Karlsruhe Institute of Technology, 76131 Karlsruhe, Germany \\ ${ }^{2}$ Department of Mechanical Engineering, The University of Melbourne, Victoria 3010, Australia
}

(Received 13 August 2019; revised 22 February 2020; accepted 22 May 2020)

We investigate the formation of subaqueous transverse bedforms in turbulent open channel flow by means of direct numerical simulations with fully resolved particles. The main goal of the present analysis is to address the question whether the initial pattern wavelength scales with the particle diameter or with the mean fluid height. A previous study (Kidanemariam \& Uhlmann, J. Fluid Mech., vol. 818, 2017, pp. 716-743) has observed a lower bound for the most unstable pattern wavelength in the range 75-100 times the particle diameter, which was equivalent to 3-4 times the mean fluid height. In the current paper, we vary the streamwise box length in terms of the particle diameter and of the mean fluid height independently in order to distinguish between the two possible scaling relations. For the chosen parameter range, the obtained results clearly exhibit a scaling of the initial pattern wavelength with the particle diameter, with a lower bound around a streamwise extent of approximately 80 particle diameters. In longer domains, on the other hand, patterns are observed at initial wavelengths in the range 150-180 times the particle diameter, which is in good agreement with experimental measurements. Variations of the mean fluid height, on the other hand, seem to have no significant influence on the most unstable initial pattern wavelength. We argue that the observed scaling with the particle diameter is due to the wake effect induced by the seeds which are formed by initially dislodged lumps of particles, in accordance with the ideas of Coleman \& Nikora (Water Resour. Res., vol. 45, 2009, W04402). Finally, for the cases with the largest relative submergence, we observe spanwise and streamwise sediment waves of similar amplitude to evolve and superimpose, leading to three-dimensional sediment patterns.

Key words: sediment transport, particle/fluid flow, turbulent boundary layers

\section{Introduction}

Sediment beds under streaming water bodies often exhibit characteristic bedforms which can significantly affect the transport properties of the flow. Among these various bedforms, transverse bedforms are usually classified as ripples, dunes or antidunes (Yalin 1977). Ripples and dunes both exhibit a roughly triangular shape with a gentle slope on 
the upstream surface and a steeper downstream face, the latter approximately inclined at the angle of repose (Best 2005). However, these two bedforms differ in their scaling properties: while ripples are small compared to the fluid height and thus their wavelength is believed to scale with the particle diameter, the height of dunes is large enough to distinctly modify the flow field over the entire flow depth. As a consequence, the amplitude of dunes should scale with the fluid height (Engelund \& Fredsoe 1982). In contrast to the remaining bedform types, antidunes exhibit a symmetric cross-section and they can, depending on the hydraulic conditions, travel in the upstream or the downstream direction. Antidunes can only form in free-surface flows, whereas ripples and dunes can form in configurations without a free surface as well, such as pipe flows (see e.g. Ouriemi, Aussillous \& Guazzelli 2009) or closed-conduit flows (see e.g. Coleman, Fedele \& Garcia 2003; Cardona Florez \& Franklin 2016).

The origin of these transverse bedforms has been found to be an instability process in which an initially flat sediment bed deforms after an initial perturbation in the flow system, leading to the formation of transverse patterns (Yalin 1977). Inglis (1949) proposed that inhomogeneities of the sediment bed such as small particle agglomerations could act as initial perturbations that trigger subsequent bedform evolution. In a more recent paper, Coleman \& Melville (1996) proposed an instability process originating in an isolated random pileup that grows with time and, once it has reached a critical height of approximately 3-4 times the particle diameter $D$, induces the generation of further pileups downstream. Coleman \& Nikora $(2009,2011)$ describe the formation of the initial bedforms as a two-stage formation process. In a first stage, random sediment patches with a typical length of (7-15)D interact on an active (but still planar) sediment bed due to sediment transport events that are believed to be related to coherent turbulent structures. Once the height of these initial random patches exceeds some threshold height, the initial disturbance stabilizes by agglomerating sediment particles, with the consequence that further downstream regular patterns of sand-wavelets form. Venditti, Church \& Bennett (2005) observed different modes of bedform initiation in their experiments depending on the flow rate and, consequently, on the Reynolds and Froude number. At lower flow rates, local defects are seen to initiate a similar formation cycle as that described by Coleman \& Melville (1996), whereas at higher flow rates, the authors observed patterns to spontaneously evolve over the entire bed, leading to a regular 'cross-hatch pattern'.

Unfortunately, up to the present date, accurate measurements of the initial bedform dimensions remain challenging. On the one hand, this is due to the very short time window in which the initial bedform evolution can be observed, and, on the other hand, due to the small height of the initial patterns of only a few particle diameters which makes them hard to detect. It is for this reason that most of the experimental studies in the last decades have focused on the description of fully developed patterns in the equilibrium state (cf. Yalin 1985; Lapotre, Lamb \& McElroy 2017), where the wavelength of the initial bedforms is typically much larger than in the initial phase (Langlois \& Valance 2007). On the other hand, only a small number of experimentalists were able to quantitatively describe the wavelength of the initial bedforms. In turbulent open channel flow, Coleman \& Melville (1996) and Coleman \& Nikora (2009) observed the initial wavelength to scale mainly with the sediment size in terms of the median grain size and to be rather unaffected by the flow conditions. Similar observations were made by Coleman et al. (2003) in turbulent closed-conduit flows as well as by Coleman \& Eling (2000) in laminar open channel flows, the latter indicating in particular that the formation of initial bedforms is not restricted to the turbulent regime and the accompanying turbulent bursts, as earlier suggested by Raudkivi (1997). Similarly, Langlois \& Valance (2007) and Cardona Florez \& Franklin (2016) both report under turbulent channel flow conditions that the initial wavelength 
depends mainly on the particle diameter, while the influence of the shear velocity and the flow conditions is rather weak. Ouriemi et al. (2009), on the contrary, measured initial wavelengths of the order of the fluid height in pipe flows, while Franklin (2008) observed a dependence of the initial wavelength on both the particle diameter and the shear velocity in experimental measurements of turbulent closed-conduit flows.

In theoretical studies based on linear stability analysis, the flow system is usually described by a simplified model for the driving flow (such as Reynolds-averaged Navier-Stokes (RANS) models or potential flow solutions) combined with a sediment bed continuity equation for the evolution of the bed (see e.g. Kennedy 1963, 1969; Charru 2006). In order to close the system of equations, a formulation for the particle flux is required. In most stability analyses, it is assumed that the particle flux and the local bed shear stress are in phase, which allows then to express the particle flux as a function of the local bed shear stress (Charru, Andreotti \& Claudin 2013). In recent studies, this assumption has been removed and additional relaxation equations are used to take into account a possible phase lag between both quantities (Charru 2006). A linear stability analysis is then performed in order to determine regions of instability in the parameter space as well as the most amplified wavelength for a given flow configuration. During the past decades, a large number of stability analyses for different flow configurations including different stabilizing and destabilizing effects has been presented for turbulent (see e.g. Richards 1980; Sumer \& Bakioglu 1984; Colombini 2004; Fourriere, Claudin \& Andreotti 2010; Colombini \& Stocchino 2011) as well as for laminar flows (see e.g. Charru \& Mouilleron-Arnould 2002; Charru \& Hinch 2006). A detailed overview of the different approaches used in linear stability analysis can be found in the reviews of Engelund \& Fredsoe (1982), Seminara (2010) and Charru et al. (2013). Recently, Zgheib \& Balachandar (2019) have presented a combined numerical-theoretical stability analysis, in which the bed shear stress for the linear stability analysis is computed by means of direct numerical simulations (DNS) in which the sediment bed is represented in a continuous and impermeable fashion.

However, despite an increasing complexity of the used models, the wavelengths predicted by most linear stability analyses still differ by more than one order of magnitude from the wavelengths observed in experiments (Langlois \& Valance 2007; Ouriemi et al. 2009). Furthermore, the observations concerning the scaling of the initial wavelengths differ markedly between the different studies. For instance, Fourriere et al. (2010) found a single region with unstable wavelengths independent of the fluid height, leading them to the conclusion that only ripples can form directly from a flat bed, while dunes develop by a ripple coarsening processes only. Colombini \& Stocchino (2011), in contrast, observed two separate regions of instability with most amplified wavelengths of the size of the fluid height and of the particle diameter, respectively, which suggests that either ripples or dunes may form out of the same instability mechanism.

It should be kept in mind that the validity of linear stability investigations is limited to the very first instances of sediment bed evolution (Coleman \& Melville 1996), i.e. the theory is not able to predict the bedform dimensions correctly, once nonlinear effects become dominant. In more recent studies, thus, weakly nonlinear analyses have been presented (e.g. Colombini \& Stocchino 2008) to take into account also nonlinear effects.

In recent years an alternative method for the investigation of sediment transport in its early stages has become available in the form of DNS featuring fully resolved particles, which allow to resolve all relevant flow scales even below the particle length scale (e.g. Kidanemariam \& Uhlmann 2014a,b; Vowinckel, Kempe \& Fröhlich 2014; Derksen 2015; Kidanemariam \& Uhlmann 2017; Vowinckel et al. 2017; Mazzuoli, Kidanemariam \& Uhlmann 2019). In a set of numerical experiments, Kidanemariam \& Uhlmann (2017) 
(henceforth denoted KU2017) have recently determined a lower bound for the minimal unstable wavelength, $\lambda_{t h}$, by reducing the streamwise domain length $L_{x}$ successively in a comparable concept as the minimal flow unit of Jiménez \& Moin (1991). It was observed that, below a threshold $L_{x}=\lambda_{t h}$, the formation of transverse bedforms is effectively hindered and a perturbed bed remains stable as a consequence of the limited domain size, even though the conditions would otherwise allow the bed to become unstable. For the considered parameter values, KU2017 found $\lambda_{t h} / D$ to be in the range $75-100$, which is equivalent to a range of $\lambda_{t h} / H_{f}=3-4$ in their cases, where $H_{f}$ denotes the mean fluid height. Since, however, the relative fluid height $H_{f} / D$ was kept constant over all simulations, it was not possible to further distinguish between the two alternative scaling relations.

The purpose of the present work is to investigate the scaling of the lower threshold for the minimal unstable wavelength. In order to be able to distinguish between the two alternative scalings, i.e. either with the particle diameter $D$ or the fluid height $H_{f}$, two new series of numerical experiments are performed in which the relative streamwise domain lengths $L_{x} / D$ and $L_{x} / H_{f}$ are varied independently. In the subsequent analysis, we investigate the influence of both length scales on the stability of the subaqueous bedforms using the newly computed DNS data.

The present manuscript is organized as follows. In $\S 2$, we briefly describe the numerical method which we use for the simulation of subaqueous sediment transport in this work. An overview over the relevant physical and numerical parameters as well as the chosen flow configurations is given in $\S 3$. Subsequently, in $\S 4$, we shortly present two different ways of defining the fluid-bed interface, depending on whether the sediment bed is analysed in a spanwise-averaged framework or in its full extent. In $\S 5$, we analyse the data obtained in the context of the two simulation series separately, focussing on the bedform geometry and its temporal evolution. A discussion of the results as well as a comparison with experimental and theoretical studies follows in $\S 6$. We conclude the study with a summary of the main findings in $\S 7$.

\section{Numerical method}

We use the same numerical method used in Kidanemariam \& Uhlmann (2014a,b, 2017) to solve the coupled fluid-solid problem. For the simulation of the fluid phase, the Navier-Stokes equations for incompressible fluids are solved numerically in the entire computational domain using a second-order finite difference scheme together with a fractional step algorithm on a uniform Cartesian grid. Time integration of the governing equations is done in a semi-implicit way, including a Crank-Nicholson scheme for the viscous terms and a low-storage three-step Runge-Kutta scheme for the nonlinear terms. The immersed boundary formulation of Uhlmann (2005) is then used to couple the flow field with the solid phase: localized force terms are introduced into the Navier-Stokes equations which impose the no-slip condition at the interface between the fluid and the solid phase. The motion of the particles is obtained by time integration of the Newton-Euler equations for rigid-body motion. The driving force and torque comprises hydrodynamic and gravitational contributions as well as those resulting from particle-particle and particle-wall contact. Owing to the fact that the characteristic time scale of particle collisions is typically several orders of magnitude smaller than those of the turbulent fluid motion, a sub-stepping method is used for the numerical time integration of the Newton-Euler equations (Kidanemariam \& Uhlmann 2014b).

Momentum exchange due to particle collisions is computed using a soft-sphere discrete-element model (DEM). In the framework of the chosen approach, two particles are 
defined as 'being in contact', if the minimal distance between their surfaces $\Delta$ falls below a force range $\Delta_{c}$. In this case, a contact force and torque act on both particles which are defined as the sum of three individual contributions, i.e. an elastic normal force, a normal damping force and a tangential frictional force. The elastic normal force is a linear function of the penetration length $\delta_{c}=\Delta_{c}-\Delta$ with a constant stiffness coefficient $k_{n}$. The normal damping force is a linear function of the normal component of the relative particle velocity between the two particles at the contact point, with a constant normal damping coefficient $c_{n}$. Similarly, the tangential frictional force is defined as a linear function of the tangential component of the relative particle velocity at the contact point, with a constant tangential friction coefficient $c_{t}$. Note that the magnitude of the tangential frictional force has an upper traction limit in the form of the Coulomb friction limit with a friction coefficient $\mu_{c}$. A detailed description of the collision model and extensive validation can be found in Kidanemariam \& Uhlmann (2014b).

For each simulation, the model thus requires the choice of the four force parameters $\left(k_{n}, c_{n}, c_{t}, \mu_{c}\right)$ as well as the force range $\Delta_{c}$. Introducing a dry restitution coefficient $\varepsilon_{d}$, which is defined as the absolute value of the ratio between the normal components of the relative velocity before and after a dry collision, allows us to relate the normal stiffness coefficient $k_{n}$ and the normal damping coefficient $c_{n}$, and to formulate the model depending on the alternative set of force parameters $\left(k_{n}, \varepsilon_{d}, c_{t}, \mu_{c}\right)$. Due to the varying particle size and submerged weight in the present simulations, the set of parameters used in Kidanemariam \& Uhlmann $(2014 a, 2017)$ has been adapted to the respective cases. The force range $\Delta_{c}$ is set equal to the uniform grid spacing $\Delta x$ for all cases with a particle diameter $D \leq 15 \Delta x$ and equal to $2 \Delta x$ for all cases with larger particles. The stiffness parameter $k_{n}$ has a value between approximately 8400 and 17000 times the submerged weight of a single particle, divided by the particle diameter in the respective case. The parameters are chosen such that the maximum overlap $\delta_{c}$ is within a few per cent of $\Delta_{c}$. The dry restitution coefficient is set to $\varepsilon_{d}=0.3$, which determines, together with the chosen value of $k_{n}$, the constant normal damping coefficient $c_{n}$. The constant tangential friction coefficient is set to the same value $c_{t}=c_{n}$. Finally, the Coulomb friction coefficient was fixed at $\mu_{c}=0.4$ except for cases $H 2 D 052$ and $H 6 D 102$, in which a slightly higher value $\mu_{c}=0.5$ was unintentionally used. In appendix A we show, however, that this difference in the limiting Coulomb friction has only a minor influence on the eventually developed bedform and that it does not affect the stability or instability of the sediment bed. In addition to the influence of $\mu_{c}$, we have also investigated the sensitivity of the sediment bed evolution on the values chosen for the dry restitution coefficient $\varepsilon_{d}$ and the force range $\Delta_{c}$. It was observed that the formation and the final shape of the developing sediment patterns are not significantly modified when changing $\varepsilon_{d}$ from its current value of 0.3 to 0.9. Similarly, it can be stated that a change of the relative force range $\Delta_{c} / \Delta_{x}$, which is achieved by refining the fluid grid by a factor of 1.5 , has no significant influence on the bedform evolution. It should be noted that the latter validation also serves as an additional grid convergence study, highlighting the fact that the chosen spatial resolution is sufficient for the purpose of capturing all relevant flow scales.

\section{Flow configuration and parameter values}

In the course of the current study, we have performed seven new independent direct numerical simulations of bedform evolution over an erodible subaqueous sediment bed in a turbulent open channel. Two additional cases from KU2017 have been included in our analysis. The studied flow configuration is shown in figure 1. As can be seen, a Cartesian coordinate system with $\boldsymbol{x}=(x, y, z)^{\mathrm{T}}$ is centred at the lower boundary of the 


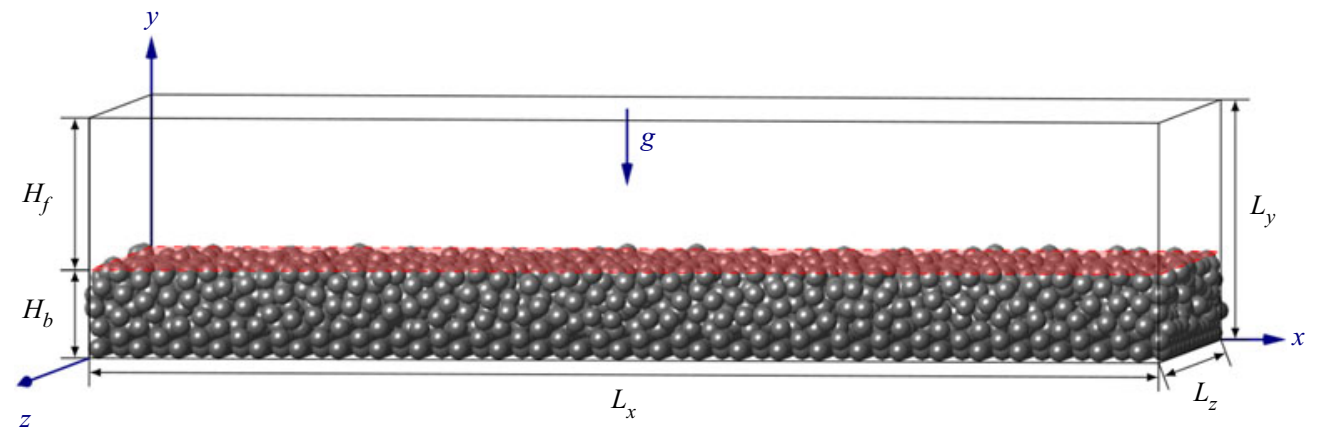

FIGURE 1. Schematic of the open channel flow configuration. Flow is in the positive $x$-direction. The computational domain is periodic along the $x$ - and $z$-directions. No-slip and free-slip boundary conditions are imposed at the bottom $(y=0)$ and top $\left(y=L_{y}\right)$, respectively.

open channel such that the $x$-, $y$ - and $z$-directions are the streamwise, wall-normal and spanwise directions, respectively. Mean flow is directed in the positive $x$-direction and gravity in the negative $y$-direction. In the streamwise and spanwise directions, periodic boundary conditions are imposed, whereas no-slip and free-slip conditions are imposed at the bottom and top planes of the channel, respectively.

As characteristic length scales, we define the mean fluid height $H_{f}$ and the mean sediment bed height $H_{b}$ as averages over both spatial directions (streamwise and spanwise) as well as over time, i.e. $H_{f}=\left\langle h_{f}\right\rangle_{x z t}$ and $H_{b}=\left\langle h_{b}\right\rangle_{x z t}$, respectively. A precise definition of $h_{f}(x, z, t)$ and $h_{b}(x, z, t)$ will be given in $\S 4.1$. Before the simulations with mobile particles are started, the sediment bed is left to settle without any fluid forces. Subsequently, turbulent flow is established while keeping the particles fixed. Finally, the particles are released at a time which will henceforth be defined as $t=0$. More details can be found in Kidanemariam \& Uhlmann (2014a). The names of the individual simulations are chosen according to their streamwise domain length in terms of the mean fluid height and in terms of the particle diameter. For instance, in case $H 4 D 052$, the relative streamwise box length is $L_{x} / H_{f} \approx 4$ and $L_{x} / D=51.2$, respectively (cf. tables 1 and 2).

In all cases, the flow is driven by a time-dependent streamwise pressure gradient, that is adjusted at each time step to ensure a constant flow rate $q_{f}$. Therefore, the bulk Reynolds number can be computed a priori as $R_{b}=u_{b} H_{f} / v$, where the bulk velocity is defined as $u_{b} \equiv q_{f} / H_{f}$. The friction Reynolds number is defined as $R e_{\tau}=u_{\tau} H_{f} / \nu$, where the friction velocity $u_{\tau}$ is computed a posteriori from the streamwise and spanwise-averaged total mean shear stress, which is composed of viscous stresses, turbulent Reynolds stresses and the stresses resulting from the fluid-particle interaction. Due to the absence of a horizontal bottom wall, we evaluate the mean friction velocity $u_{\tau}$ at the location of the mean fluid-bed interface $y=H_{b}$, which can be interpreted as a virtual wall. For a more detailed description of the determination of the shear stress distribution, the reader is referred to KU2017.

From dimensional analysis, it can be concluded that, by adding sediment to a turbulent flow, the parameter space increases and in total, four non-dimensional numbers are required to fully describe a given system. In addition to $R e_{b}$, we choose the density ratio $\rho_{p} / \rho_{f}$, the non-dimensional length scale $H_{f} / D$ as well as the Galileo number $G a=u_{g} D / v$, which expresses the ratio between gravity and viscous forces, where the gravitational velocity scale is $u_{g}=\sqrt{\left(\rho_{p} / \rho_{f}-1\right)|\boldsymbol{g}| D}$.

In the present simulations, we set the density ratio at $\rho_{p} / \rho_{f}=2.5$ which is comparable to the values reported for glass in water. To allow pattern formation, the non-dimensional 


\begin{tabular}{lccccccccc}
\hline Case & $R e_{b}$ & $R e_{\tau}$ & $\rho_{p} / \rho_{f}$ & \multicolumn{1}{c}{$G a$} & $D^{+}$ & $H_{f} / D$ & $H_{b} / D$ & $\theta$ & Source \\
$-H 2 D 052$ & 3011 & 242.2 & 2.5 & 28.37 & 9.59 & 25.25 & 13.15 & 0.11 & Present \\
$-H 4 D 052$ & 3011 & 281.0 & 2.5 & 56.74 & 22.67 & 12.40 & 6.80 & 0.16 & Present \\
$-H 6 D 052$ & 3011 & 311.1 & 2.5 & 104.24 & 39.62 & 7.86 & 4.94 & 0.14 & Present \\
$-H 6 D 077$ & 3011 & 286.1 & 2.5 & 56.74 & 23.18 & 12.34 & 6.86 & 0.17 & Present \\
$-H 6 D 102$ & 3011 & 279.4 & 2.5 & 43.61 & 17.10 & 16.33 & 9.27 & 0.15 & Present \\
$-H 6 D 154$ & 3011 & 303.0 & 2.5 & 28.37 & 11.90 & 25.47 & 12.93 & 0.18 & $H 6$ in KU2017 \\
$-H 4 D 102^{1,2,3}$ & 3011 & 267.3 & 2.5 & 28.37 & 10.63 & 25.15 & 13.25 & 0.14 & $H 4^{1,2,3}$ in KU2017 \\
$-H 2 D 102^{1}$ & 5013 & 360.5 & 2.5 & 16.78 & 7.14 & 50.53 & 13.47 & 0.18 & Present \\
$-H 2 D 102^{2}$ & 5013 & 358.5 & 2.5 & 20.06 & 7.09 & 50.59 & 13.41 & 0.13 & Present
\end{tabular}

TABLE 1. Physical parameters of the simulations. $R e_{b}, R e_{\tau}$ and $D^{+}$are the bulk Reynolds number, the friction Reynolds number and the particle Reynolds number, respectively. The density ratio $\rho_{p} / \rho_{f}$ and the Galileo number $G a$ are imposed in each simulation, whereas the Shields number $\theta$, the relative submergence $H_{f} / D$ and the relative sediment bed height $H_{b} / D$ are computed a posteriori (cf. table 2 ). The last column provides information about the source of the listed cases, distinguishing between simulations that have been computed in the course of the current study (present) and cases that are from Kidanemariam \& Uhlmann (2017) (KU2017). It should be further mentioned that the physical parameters presented for case $H 4 D 102^{1,2,3}$ have been averaged over the three individual simulations. A list of the physical parameters for each individual run can be found in KU2017.

$\begin{array}{lccccccc}\text { Case } & {\left[L_{x} \times L_{y} \times L_{z}\right] / D} & L_{x} / H_{f} & D / \Delta x & \Delta x^{+} & N_{p} & T_{o b s} / T_{b} & T_{o b s}^{s} / T_{b} \\ -H 2 D 052 & 51.2 \times 38.4 \times 76.8 & 2.03 & 10 & 0.96 & 43730 & 742 & 691 \\ -H 4 D 052 & 51.2 \times 19.2 \times 38.4 & 4.13 & 20 & 1.13 & 9923 & 497 & 177 \\ -H 6 D 052 & 51.2 \times 12.8 \times 25.6 & 6.52 & 30 & 1.32 & 5086 & 879 & 405 \\ -H 6 D 077 & 76.8 \times 19.2 \times 38.4 & 6.22 & 20 & 1.16 & 14954 & 918 & 486 \\ -H 6 D 102 & 102.4 \times 25.6 \times 51.2 & 6.27 & 15 & 1.14 & 44163 & 618 & 235 \\ -H 6 D 154 & 153.6 \times 38.4 \times 76.8 & 6.04 & 10 & 1.19 & 127070 & 918 & 513 \\ -H 4 D 102^{1,2,3} & 102.4 \times 38.4 \times 76.8 & 4.07 & 10 & 1.06 & 86645 & 401 / 400 / 853 & 88 / 84 / 542 \\ -H 2 D 102^{1} & 102.4 \times 64.0 \times 76.8 & 2.03 & 10 & 0.71 & 86645 & 736 & 393 \\ -H 2 D 102^{2} & 102.4 \times 64.0 \times 76.8 & 2.02 & 10 & 0.71 & 86645 & 509 & 252\end{array}$

TABLE 2. Numerical parameters of the simulations. The computational domain has dimensions $L_{i}$ in the $i$ th direction and is discretized using a uniform grid with mesh width $\Delta x, \Delta x^{+}$ denoting the grid width in wall units. $N_{p}$ is the total number of particles in the respective case. The time is scaled in bulk time units $T_{b}=H_{f} / u_{b} . T_{o b s}$ is the total observation time of each simulation starting from the release of the moving particles. Time-dependent physical and numerical parameters in tables 1 and $2\left(R e_{\tau}, D^{+}, H_{f}, H_{b}, \theta, \Delta x^{+}\right)$are computed as an average over a final time interval $T_{o b s}^{s}$.

boundary shear stress, expressed by the Shields number $\theta=u_{\tau}^{2} / u_{g}^{2}=\left(D^{+} / G a\right)^{2}$, is chosen larger than the critical value for incipient sediment motion. Note that quantities marked as $(\cdot)^{+}$are scaled in wall units. In turbulent flows, the critical Shields number has been 
observed to be in a range $\theta_{c}=0.03-0.05$, slightly depending on the Galileo number (Soulsby \& Whitehouse 1997; Wong \& Parker 2006; Franklin \& Charru 2011).

The non-dimensional mean fluid height $H_{f} / D$ is varied in the different simulations to elucidate the relevant length scales that dominate the scaling of subaqueous bedforms by either increasing the particle diameter or the mean fluid height while keeping the same dimensions for the domain. As a consequence, the number of fully resolved particles lies in a range between $O\left(10^{3}\right)$ in the case with the largest particles and up to $O\left(10^{5}\right)$ in the case with the smallest particles.

Note that, in all cases, the dimensions of the computational domain are sufficiently large to allow self-sustained turbulence. In particular, the cases with the shortest streamwise and spanwise dimensions (scaled in viscous lengths) are case $H 2 D 052$ with $L_{x}^{+} \approx 490$ and case $H 2 D 102^{2}$ with $L_{z}^{+} \approx 555$, respectively. By comparison, Jiménez \& Moin (1991) report the dimensions of their minimal flow unit as $L_{x}^{+} \approx 250-350$ and $L_{x}^{+} \approx 100$, respectively.

In $\S 5$, we will analyse the two simulation series separately: while in the first series (including cases $H 6 D 052, H 6 D 077, H 6 D 102$ and $H 6 D 154$ ), the $L_{x} / D$ ratio has been varied keeping $L_{x} / H_{f}$ constant, $L_{x} / D$ is fixed in the second series (including cases $H 4 D 102^{1,2,3}, H 2 D 102^{1}$ and $H 2 D 102^{2}$ ) and $L_{x} / H_{f}$ is varied instead. It should be noted that we have performed two additional simulations, $H 2 D 052$ and $H 4 D 052$, which do not fit into either of these two series. Therefore, we discuss the results obtained in these latter cases in $\S 6$ only.

\section{Extraction of bedform dimensions}

For the following definition of the fluid-bed interface, we will consider the domain as composed of two distinct regions that are a lower particle-dominated region, hereafter termed the sediment bed, and the overlaying fluid-dominated region. Hence, the wall-normal dimension of the channel $L_{y}$ can be written as the sum of the instantaneous local height of the fluid phase $h_{f}(x, z, t)$ and that of the sediment bed $h_{b}(x, z, t)$, viz.

$$
L_{y}=h_{b}(x, z, t)+h_{f}(x, z, t) .
$$

In the chosen Cartesian coordinate system, the wall-normal location of the fluid-bed interface is identical to the value of the function $h_{b}(x, z, t)$. In the following, we will present two different approaches to define the instantaneous fluid-bed interface. The first method defines the fluid-bed interface through a spanwise averaging, whereas the second extracts the sediment bed as a two-dimensional surface.

\subsection{Definition and analysis of the spanwise-averaged fluid-bed interface}

Assuming statistical homogeneity of the observed system, (4.1) can be averaged in the spanwise direction as

$$
L_{y}=\left\langle h_{b}\right\rangle_{z}(x, t)+\left\langle h_{f}\right\rangle_{z}(x, t) .
$$

The spanwise-averaged sediment bed height $\left\langle h_{b}\right\rangle_{z}$ is determined depending on a threshold for the solid volume fraction. Since the method is presented in detail in our previous works (Kidanemariam \& Uhlmann 2014a, 2017), we restrict ourselves to a short summary of the most important points. First, a solid-phase indicator function $\phi_{p}(\boldsymbol{x}, t)$ is defined, which attains the value of unity for Eulerian grid points located inside the particle domain $\Omega_{p}$ and zero elsewhere. Second, the spanwise-averaged sediment bed height $\left\langle h_{b}\right\rangle_{z}$ is defined 
as the wall-normal location, at which the spanwise-averaged solid indicator function $\left\langle\phi_{p}\right\rangle_{z}$ attains a threshold of $\left\langle\phi_{p}\right\rangle_{z}^{\text {thresh }}=0.1$ (Kidanemariam \& Uhlmann 2014b), i.e.

$$
\left\langle h_{b}\right\rangle_{z}(x, t)=y, \quad \mid\left\langle\phi_{p}\right\rangle_{z}(x, y, t)=\left\langle\phi_{p}\right\rangle_{z}^{\text {thresh }} .
$$

The spanwise-averaged fluid height $\left\langle h_{f}\right\rangle_{z}$ is computed using relation (4.2). Eventually, we perform another decomposition in the streamwise direction, similar to the spanwise decomposition used in (4.2). The resulting expression

$$
\left\langle h_{b}\right\rangle_{z}(x, t)=\left\langle h_{b}\right\rangle_{x z}(t)+\left\langle h_{b}\right\rangle_{z}^{\prime}(x, t)
$$

divides the spanwise-averaged interface in an instantaneous mean height $\left\langle h_{b}\right\rangle_{x z}(t)$ and a fluctuation $\left\langle h_{b}\right\rangle_{z}^{\prime}(x, t)$ with respect to the former. In the following, the fluctuation will form the basis for the analysis of the bedform evolution. The size and shape of two-dimensional transverse patterns are usually quantified by the pattern length and height. Over the last decades, a variety of different approaches has been developed to define these length scales. An overview over some of these methods is presented in Coleman \& Nikora (2011). In the current work, we choose a statistical definition for the pattern height as well as for the wavelength of the bedforms (Langlois \& Valance 2007). As a measure for the pattern height, we use the root mean square of the sediment bed height fluctuation

$$
\sigma_{h}(t)=\sqrt{\left\langle\left\langle h_{b}\right\rangle_{z}^{\prime}(x, t) \cdot\left\langle h_{b}\right\rangle_{z}^{\prime}(x, t)\right\rangle_{x}}
$$

In order to determine the average pattern wavelength, let us define the instantaneous two-point correlation coefficient of the sediment bed height fluctuation

$$
R_{h}(\delta x, t)=\left\langle\left\langle h_{b}\right\rangle_{z}^{\prime}(x, t) \cdot\left\langle h_{b}\right\rangle_{z}^{\prime}(x+\delta x, t)\right\rangle_{x} / \sigma_{h}^{2}(t),
$$

where $\delta x$ expresses the streamwise separation length between two points. With increasing $\delta x$, the evolution of $R_{h}(\delta x, t)$ at a given time $t$ behaves similarly to a damped oscillation curve around a zero mean. The global (and first) minimum of the curve occurs at a separation $\delta x_{\min }$, i.e.

$$
R_{h}\left(\delta x_{\min }, t\right) \leq R_{h}(\delta x, t) \quad \forall \delta x \in\left[0, L_{x} / 2\right]
$$

The mean wavelength is finally obtained as twice this separation length, i.e. $\lambda_{h}(t)=2 \delta x_{\text {min }}$.

\subsection{Definition and analysis of the two-dimensional fluid-bed interface}

The spanwise-averaged definition and analysis, as described in the previous section, is restricted to a fluid-bed interface that is statistically homogeneous in the spanwise direction. However, in the general case, this procedure may be not adequate to describe possible three-dimensional sediment patterning.

Here, we use a criterion based on determining the top-layer particles of the fluid-bed interface. In classical morphodynamics, the sediment bed is distinguished from the bedload and suspended load (Bagnold 1956; van Rijn 1984). Following this classification, we have developed an algorithm which sorts out the latter two categories, leaving only the particles that are part of the sediment bed itself. First, single suspended particles are detected based on the wall-normal collision force component $F_{c, y}$. While particles inside the bed are exposed to a permanent wall-normal contact force of the order of their 
submerged weight $F_{w}=\left(\rho_{p}-\rho_{f}\right) \pi D^{3} / 6|g|$, single suspended particles are typically not in contact with surrounding particles and, accordingly, only a small contact force acts on them. Thus, particles that are only exposed to a negligible wall-normal contact force will not be considered as 'bed particles' (where we have used a threshold $\left|F_{c, y}\right| / F_{w}<10^{-5}$ in practice, the precise value of which was not found to have any significant consequence for the extracted bed height).

The remaining particles include both the actual sediment bed and the bedload layer. Particles inside the latter are in motion close to the bed, such that they lose contact to the bed only for short time intervals (Yalin 1977). In order to separate the bedload transport particles from the bed particles, a criterion based upon the particle speed is employed, eliminating all particles which exceed a threshold value. The threshold value can be chosen by defining a non-dimensional particle Shields number $\theta_{p}=\left(\left|\boldsymbol{u}_{p}\right| / u_{g}\right)^{2}$ with the norm of the particle velocity $\left|\boldsymbol{u}_{p}\right|$ and the gravitational velocity scale $u_{g}$. The sediment bed particles are then found as the set of all particles for which $\theta_{p} \leq \theta_{c}$. Here, we choose as the critical value $\theta_{c}=0.05$ (Wong \& Parker 2006). Note that the particle velocity criterion also eliminates suspended particle pairs which might instantaneously experience collision forces above the chosen threshold of the wall-normal contact force.

The fluid-bed interface is then defined through a three-dimensional $\alpha$-shape (Edelsbrunner \& Mücke 1994) enclosing the set of sediment bed particles. This enclosing surface can be thought of as a generalization of a convex hull, with an imposed radius $\alpha$ (here taken as 1.1 times the particle diameter) that defines the length scale above which non-convexity is allowed. The fluid-bed interface is then made up of those triangular faces of the $\alpha$-shape which have an outward-pointing face normal with a positive $y$-component. The data consist of a function $h(x, z)$ which is sampled at the projection of the vertex points of the surface triangulation upon the $(x, z)$-plane at a given time. In a final step, this function $h(x, z)$ is interpolated to a uniform Eulerian grid with a mesh width equal to one particle diameter and the result is smoothed using a two-dimensional box filter with a width of 5 particle diameters.

For the analysis of this two-dimensional interface, let us extend our definition of the root mean square of the sediment bed height fluctuation, as defined in (4.5), to the multidimensional case in the following form:

$$
\sigma_{h}^{2 D}(t)=\sqrt{\left\langle h_{b}^{\prime}(x, z, t) \cdot h_{b}^{\prime}(x, z, t)\right\rangle_{x z}},
$$

including the two-dimensional sediment bed height fluctuation $h_{b}^{\prime}(x, z, t)$ with respect to the streamwise- and spanwise-averaged mean $\left\langle h_{b}\right\rangle_{x z}(t)$.

\section{Results}

\subsection{Variation of the particle diameter}

In a first series, the influence of the particle diameter $D$ on the minimal unstable wavelength $\lambda_{t h}$ at a given parameter point is investigated. To this end, a series of four simulations with different particle size is performed, in which the particle diameter $D$ is successively increased from $D / H_{f} \approx 0.04$ in case $H 6 D 154$ to $D / H_{f} \approx 0.13$ in case $H 6 D 052$. It should be stressed that, in all four simulations, the streamwise domain length $L_{x}$ is almost constant in the range $(6.04-6.52) H_{f}$ and thus clearly above the critical value observed in KU2017. Note that case $H 6 D 154$ with the smallest particle diameter is identical to case $H 6$ presented in KU2017, in which a single bedform has been observed to evolve and to eventually reach a quasi-equilibrium state. 


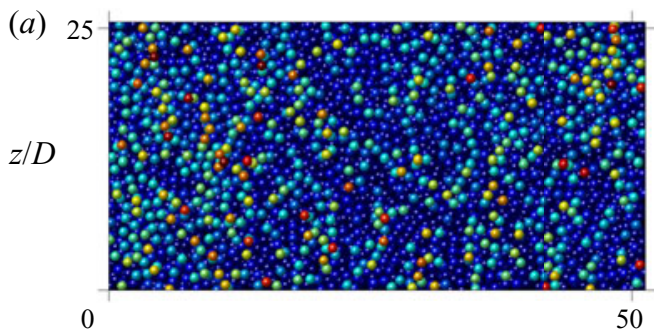

(b)
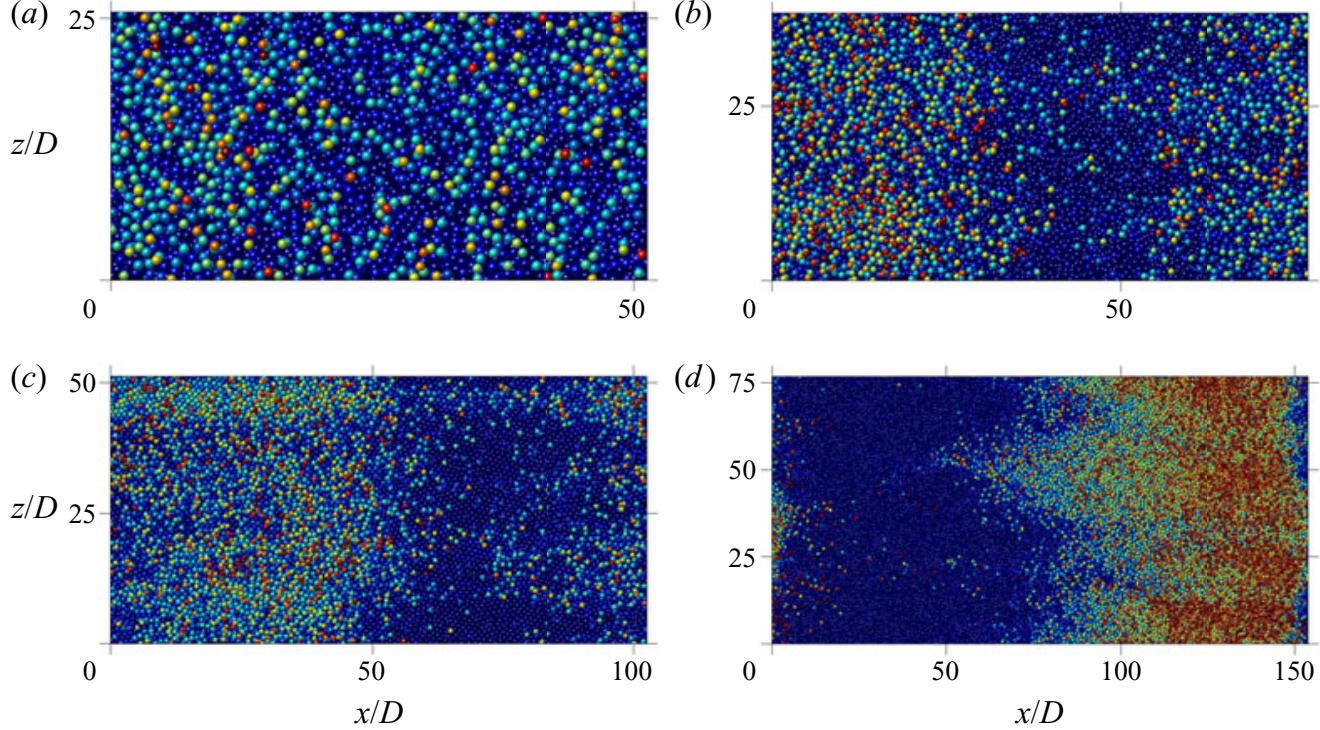

(d)
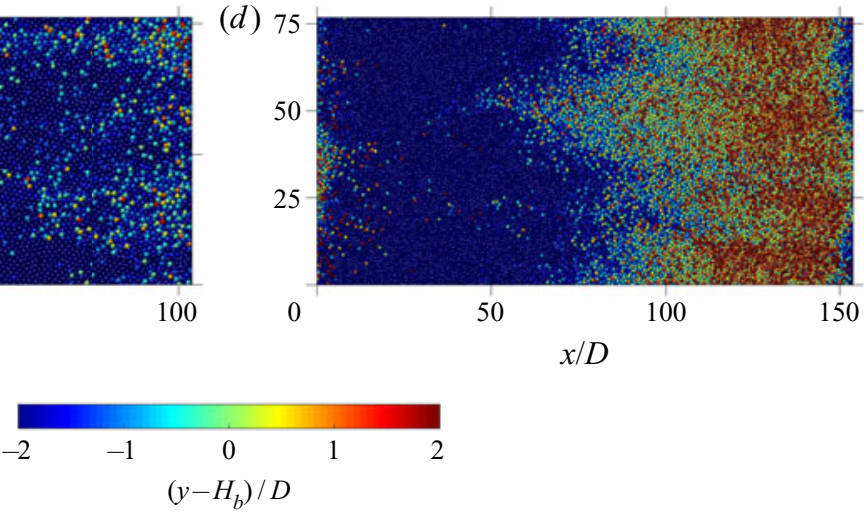

FIGURE 2. Instantaneous snapshots of the sediment bed of the cases varying the particle diameter, seen from the top of the channel. Particles are coloured depending on the wall-normal location of their centre, as shown in the global colour code. The bedforms shown in the figures have been observed at $t \approx 300 T_{b}$ for all cases: (a) H6D052, (b) H6D077, (c) H6D102, (d) H6D154. See also the supplementary movies available at https://dx.doi.org/10.4121/uuid: 7eb6a0be-ff83-4883-9d99-31daaa6a2863.

Figure 2 shows instantaneous snapshots of the sediment bed in the different cases after a simulation period of approximately 300 bulk time units. It is seen that in case $H 6 D 052$, no transverse pattern has formed. Instead, the bed has remained essentially flat and eroded sediment seems to distribute over the entire channel length and width, without accumulating in specific locations. In particular, case $H 6 D 052$ does not exhibit a similar regular pattern of streamwise aligned alternating ridges and troughs as those KU2017 found in the stable sediment bed of their case $H 3$. On the other hand, the remaining new cases with a domain length $L_{x} \geq 76.8 D$ are unstable, each featuring one single transverse bedform with an initial wavelength of the order of the streamwise box length. This indicates that, at the given parameter point, there is indeed a lower limit for $\lambda_{t h}$, which depends on the particle diameter and which is found in the range (51.2-76.8)D. As can already be inferred from the top-view visualizations of the sediment bed (cf. figure 2), the shape of those bedforms which do emerge differs, in particular in the vicinity of the threshold.

In order to provide a quantitative description of these patterns, we will now discuss various geometrical measures. First, we will focus on the pattern height evolution by studying the root mean square sediment bed height fluctuation $\sigma_{h}$, which can be seen as a measure for the inhomogeneity of the sediment bed height (Kidanemariam \& Uhlmann 2014a, 2017; Zgheib et al. 2018). In a case in which no transverse bedforms evolve, $\sigma_{h}$ will show some fluctuations due to random uncorrelated bed undulations, but it will remain bounded by a small value in the course of the simulation. A sediment bed that shows such 


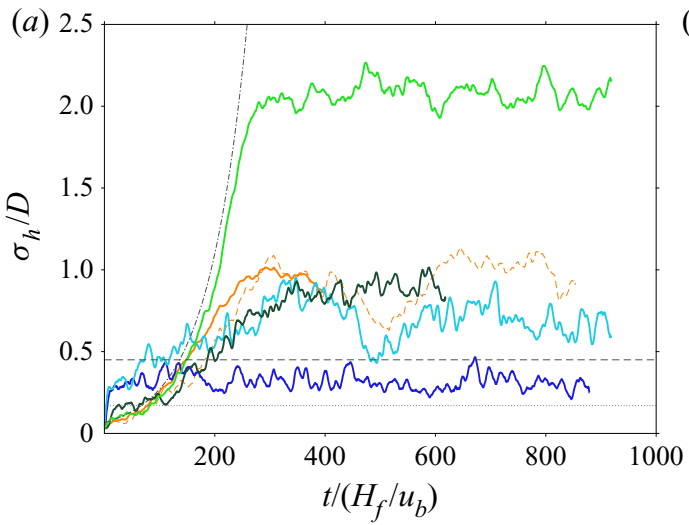

H6D052

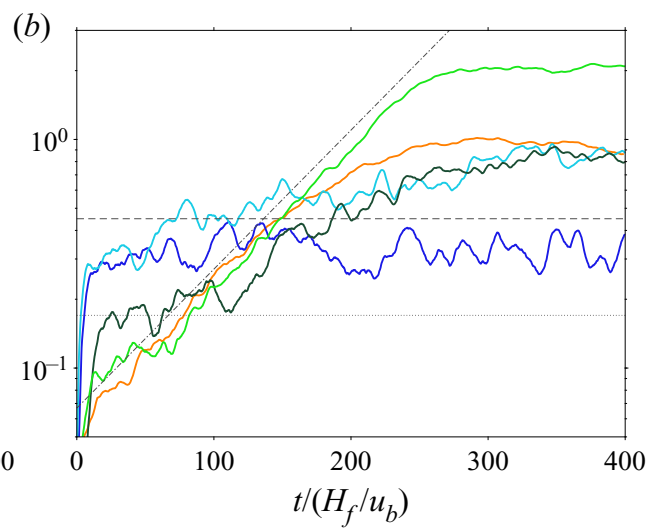

$H 6 D 154$

$H 4 D 102^{1,2,3}$

$H 4 D 102^{3}$

FIGURE 3. (a) Time evolution of the root mean square of the bedform amplitude in cases $H 6 D 052, H 6 D 077, H 6 D 102$ and $H 6 D 154$ normalized by the particle diameter $\sigma_{h} / D$. Time is scaled in bulk time units $T_{b}$. The data of case $H 4 D 102^{1,2,3}$ are presented as an ensemble average over the three simulations. Additionally, the individual evolution of run $H 4 D 102^{3}$ is presented. The horizontal dotted and dashed lines indicate values reported by Coleman \& Nikora (2009) for a 'static plane bed' $\left(\sigma_{h} \approx 0.17 D\right)$ and 'mobile sediments on planar but active beds' $\left(\sigma_{h} \approx 0.40-0.50 D\right.$, here $\left.\sigma_{h} \approx 0.47 D\right)$. The dashed-dotted line represents the exponential curve $\sigma_{h} / D=0.0668 \exp \left(0.0140 t / T_{b}\right)$ found by KU2017 as the best fit for the initial growth of $\sigma_{h}$ in their cases (including the present case $H 6 D 154$ ). Mean values of $\sigma_{h}$ averaged over the final time interval $T_{o b s}^{s}$ are as follows: $H 6 D 052:\left\langle\sigma_{h}\right\rangle_{t} / D \approx 0.30, H 6 D 077:\left\langle\sigma_{h}\right\rangle_{t} / D \approx 0.67, H 6 D 102$ : $\left\langle\sigma_{h}\right\rangle_{t} / D \approx 0.89, H 6 D 154:\left\langle\sigma_{h}\right\rangle_{t} / D \approx 2.08, H 4 D 102^{1,2,3}:\left\langle\sigma_{h}\right\rangle_{t} / D \approx 0.96$. (b) Same data as $(a)$, but represented in semi-logarithmical scale.

evolution will be classified as stable. In unstable systems, on the other hand, $\sigma_{h}$ will grow more or less monotonically during the initial phase of the simulation and, in particular, it will exceed the aforementioned threshold that bounds the stable cases. Figure 3 shows the time evolution of $\sigma_{h}$. After a short initial transient of a few bulk time units during which the chaotic particle motion leads to small finite values of $\sigma_{h}$, we observe that in all cases with $L_{x} \geq 76.8 D, \sigma_{h}$ increases with time starting at $t \approx 20 T_{b}$, indicating that the chosen relative box length is sufficiently long to cover at least one unstable mode and thus to allow the bed to evolve transverse patterns. On the contrary, $\sigma_{h}$ in case $H 6 D 052$ does not exhibit any substantial growth period. Instead, it remains around a value of $\sigma_{h} \approx 0.3 D$, only featuring fluctuations of small amplitude, bounded by an upper value of approximately $0.47 \mathrm{D}$. This value is in good agreement with the results of Coleman \& Nikora (2009), who observed 'mobile sediments on planar but active bed' for $\sigma_{h}$ in the range $(0.40-0.50) D$. Unstable bedforms are usually observed to run through different phases of bedform evolution (KU2017). During an initial growth period, the bed increases exponentially as predicted by linear stability theory. After some time, nonlinear contributions become relevant and let the bed height tend to its quasi-steady equilibrium value (Charru et al. 2013). KU2017 observed that all their unstable cases exhibited an exponential growth at a very similar growth rate, apparently independent of the streamwise box length of the respective case. They found an exponential function

$$
\sigma_{h} / D=A \exp \left(B t / T_{b}\right)
$$




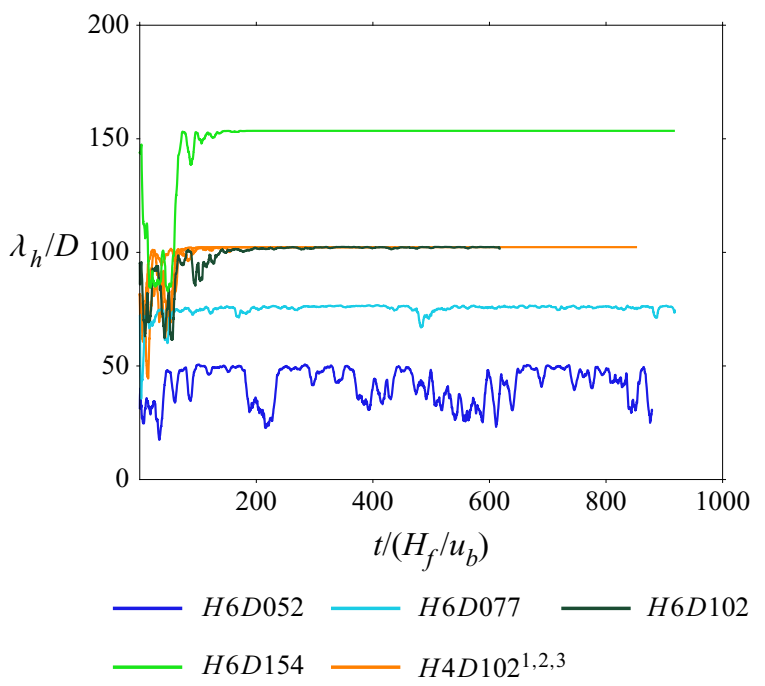

FIGURE 4. Time evolution of the mean wavelength of the sediment bed height in cases $H 6 D 052, H 6 D 077, H 6 D 102$ and $H 6 D 154$ normalized by the particle diameter $\lambda_{h} / D$. Colour coding similar to figure 3 .

with amplitude $A=0.0668$ and growth rate $B=0.0140$ to best fit the initial increase of $\sigma_{h}$ with time over an interval of approximately 150 bulk time units. In contrast, the temporal evolution in the first instances of the current unstable cases strongly varies from case to case. While the initial growth of case $H 6 D 154$ follows the above described exponential function of KU2017, the remaining two cases $H 6 D 102$ and $H 6 D 077$ grow more slowly. In the subsequent phase, case $H 6 D 154$ and $H 6 D 102$ attain what could be called as asymptotic state after approximately 280 and 350 bulk time units, respectively. Case $H 6 D 077$ exhibits stronger oscillations with higher amplitude compared to the previous two cases, a behaviour similar to that of case $H 4 D 102^{3}$ from KU2017. Averaging $\sigma_{h}$ over the final time interval $T_{\text {obs }}^{s}$ leads to values of $\left\langle\sigma_{h}\right\rangle_{t} / D \approx 2.08,\left\langle\sigma_{h}\right\rangle_{t} / D \approx 0.89$ and $\left\langle\sigma_{h}\right\rangle_{t} / D \approx 0.67$ for cases $H 6 D 154, H 6 D 102$ and $H 6 D 077$, respectively. It is remarkable that the mean values in the final interval differ by more than a factor of two between cases $H 6 D 154$ and $H 6 D 102$, which possess a comparable relative domain length of $L_{x} / H_{f} \approx 6$. On the other hand, cases $H 6 D 102$ and $H 4 D 102^{1,2,3}$ attain very similar values of $\left\langle\sigma_{h}\right\rangle_{t} / D \approx 0.89$ and $\left\langle\sigma_{h}\right\rangle_{t} / D \approx 0.96$, respectively, although case $H 4 D 102^{1,2,3}$ has a smaller relative box length $L_{x} / H_{f} \approx 4$. This indicates that the attained mean value of $\sigma_{h}$ in the final interval mainly depends on the chosen $L_{x} / D$ ratio, whereas it is not very sensitive to a variation of the mean fluid height $H_{f}$.

Figure 4 shows the time evolution of the mean pattern wavelength $\lambda_{h}$. It can be observed that the chosen mean wavelength for all cases with $L_{x} \geq 76.8 \mathrm{D}$ settles, after some fluctuation in the first 100-200 bulk time units, at the maximum possible wavelength, i.e. $\lambda_{h}=L_{x}$, and maintains this value until the end of the simulation. KU2017 observed a similar evolution of the mean wavelength settling at $\lambda_{h}=L_{x}$ for their shorter cases up to a box length $L_{x}=179.2 D$. The current observations further support their findings that these systems cannot freely choose their initial wavelength, but that they are constrained by the limitation of the streamwise domain size. As a consequence, the system chooses the maximum possible unstable wavelength as the dominant one, which, however, is not necessarily the same as the one it would choose in a system without spatial limitations (cf. the very long box with $L_{x} / H_{f}=48$ simulated by KU2017). In contrast to the observed 

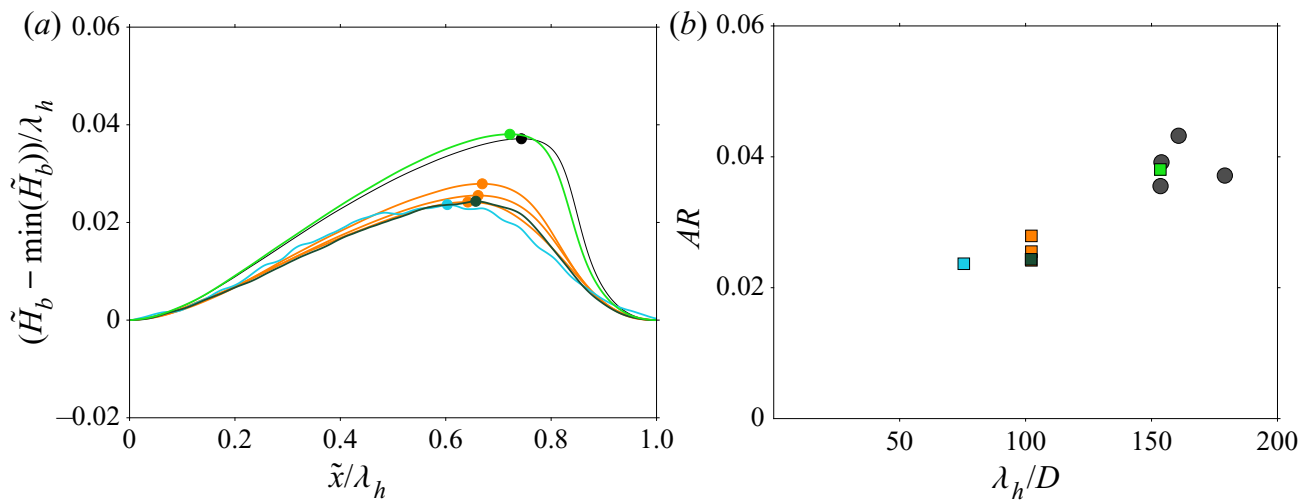

$H 6 D 077-H 6 D 154$ $H 6 D 102$ $H 4 D 102^{1,2,3}$

FIGURE 5. (a) Mean two-dimensional profile of the sedimentary patterns in cases $H 6 D 077, H 6 D 102, H 6 D 154$ and $H 4 D 102^{1,2,3}$, averaged over the time period $T_{o b s}^{s}$ at the end of each simulation. The filled circles indicate the location, where each profile attains its maximal value. Note that for the sake of visualization, the vertical and the horizontal axes are shown in different scales, i.e. the aspect ratio is exaggerated. The black line shows the same quantity for case $H 7$ from KU2017, with $L_{x} / D=179.2$ and $L_{x} / H_{f}=7.08$. (b) Aspect ratio $(A R)$ of the mean two-dimensional profiles presented in $(a)$ as a function of the mean wavelength $\lambda_{h}$ (averaged over the final time interval $T_{o b s}^{s}$ ). Round symbols represent case $H 7$ as well as the three individual runs of case $H 12$ from KU2017.

unstable cases, $\lambda_{h}$ in case $H 6 D 052$ jumps between the first three harmonics $\lambda_{1}=L_{x}$, $\lambda_{2}=L_{x} / 2$ and $\lambda_{3}=L_{x} / 3$ for the entire observation interval. This behaviour is caused by random uncorrelated bed disturbances and it indicates that the system is not able to develop a pattern at a finite wavelength.

In the following, we will investigate the fully developed spanwise-averaged profile of the sediment pattern along the streamwise direction. To this end, we compute the phase-averaged bed height $\tilde{H}_{b}(\tilde{x})$ as defined in KU2017. For this purpose we use a constant pattern migration velocity $u_{D}$ which is determined from a linear fit of the space-time correlation. Phase averaging is performed over the final time interval $T_{o b s}^{s}$ (as indicated in table 2). We further introduce the aspect ratio $A R$ and the degree of asymmetry $L R$ of the pattern as (KU2017)

$$
A R=H_{D} / \lambda_{h}, \quad L R=l_{D} / \lambda_{h},
$$

where $H_{D}=\max \left(\tilde{H}_{b}\right)-\min \left(\tilde{H}_{b}\right)$ is the pattern height, $l_{D}$ is the streamwise distance from the crest to the neighbouring downstream trough and $\lambda_{h}$ is the mean wavelength.

Figure 5 shows the mean pattern profile averaged over the final time interval of all simulations as well as the attained aspect ratio of the bedforms. It can be seen that the profiles of cases with $L_{x} / D \geq 153.6$ almost collapse upon a self-similar profile exhibiting a strong asymmetry $(L R \approx 0.28)$ and an aspect ratio of $A R \approx 0.037$ (KU2017). Some experimental studies report higher values for the steady-state aspect ratio, i.e. $A R \approx 0.045$ (Fourriere et al. 2010), $A R \approx 0.05$ (Andreotti \& Claudin 2013) and $A R \approx 0.067$ (Charru et al. 2013). KU2017 explain their lower value with the fact, that in their cases, 'the "natural" steady-state regime is not yet reached' and thus, a larger aspect ratio might be attained in a later phase, whereas the experimental data reflect the long-time state of the system which in those cases has evolved for at least one order of magnitude longer 
times. On the other hand, the pattern profiles in cases $H 6 D 102$ and $H 4 D 102^{1,2,3}$ with $L_{x}=102.4 D$ are characterized by a smaller aspect ratio of $A R \approx 0.024-0.028$ and a more symmetric shape $(L R \approx 0.33-0.036$ ). Important to note is, however, that all four profiles with $L_{x}=102.4 D$ are again nearly self-similar, despite the fact that $L_{x} / H_{f}$ varies by up to a factor of 1.5. This is another indication that the pattern shape is mainly controlled by the particle diameter $D$, but almost unaffected by the mean fluid height $H_{f}$. Further reducing the relative domain length to a value $L_{x} / D=76.8$ in case $H 6 D 077$ leads to an even more symmetric profile $(L R \approx 0.41)$, whereas the aspect ratio still attains a value $A R \approx 0.024$, comparable to the cases with $L_{x}=102.4 D$. An additional observation in case $H 6 D 077$ is that, in contrast to the previous cases, the curve representing the pattern profile is not smooth, but exhibits wavy disturbances. This is a direct consequence of the lower amount of particles in case $H 6 D 077$, which leads to stronger disturbances of the spanwise-averaged pattern profile.

\subsection{Variation of the mean fluid height}

In order to study the dependence of the minimal unstable wavelength $\lambda_{t h}$ on the mean fluid height $H_{f}$, a second set of three simulations will be analysed in the following, including case $H 4 D 102^{1,2,3}$ of KU2017 as well as two new simulations $H 2 D 102^{1}$ and $H 2 D 102^{2}$. All three cases have the same initial sediment bed configuration and the streamwise and spanwise box dimensions match. However, in cases $H 2 D 102^{1}$ and $H 2 D 102^{2}, H_{f}$ has been increased by a factor of two compared to case $H 4 D 102^{1,2,3}$. Consequently, the bulk and friction Reynolds numbers are higher in the former two cases (cf. table 1). In cases $H 2 D 102^{1}$ and $H 2 D 102^{2}$, two different values have been chosen for the Shields number, i.e. $\theta=0.18$ in the former and $\theta=0.13$ in the latter, by adjusting the Galileo number, while the remaining parameters are identical. The dimensions of the channel configuration in the new simulations have been chosen in such a way, that their relative domain length $\left(L_{x} / H_{f} \approx 2\right)$ lies below the lower bound for the most unstable wavelength as initially reported by KU2017. As a consequence, none of the two cases should allow the sediment bed to become unstable and transverse patterns to evolve, if the minimal unstable wavelength scales with the mean fluid height. Note, however, that in the study of KU2017 the values of $L_{x} / D$ and $L_{x} / H_{f}$ were not varied independently, and, therefore, it was not possible to distinguish between the two alternative scaling relations. Here, we are now in a position to do this.

Figures 6 and 7 show selected instantaneous snapshots of the particle bed evolution for the new cases $H 2 D 102^{1}$ and $H 2 D 102^{2}$, respectively. The sediment bed surface of case $H 2 D 102^{1}$ is deformed, showing streamwise and spanwise elongated crestlines. In some phases, the system alternates between patterns at either of the two orientations (cf. figure $6 b, c$ ), whereas in figure 6(a), for instance, streamwise and spanwise oriented crestlines appear at the same time. Similarly, the sediment bed of case $H 2 D 102^{2}$ exhibits one crest line parallel and one perpendicular to the mean flow direction in figure $7(a)$. In contrast, a later snapshot in figure 7(b) shows a three-dimensional bedform with a horseshoe-like shape with horns on both sides pointing downstream. This pattern resembles in its geometry a barchan dune (Franklin \& Charru 2011). In the last snapshot provided in figure 7(c) a diagonal crest line has evolved crossing the whole extent of the $(x, z)$-plane from the lower left to the upper right corner. These observations represent a marked difference between the systems with larger clear fluid height $\left(H_{f} / D \approx 50\right)$ and those with smaller relative submergences $\left(H_{f} / D \approx 25\right)$. In the latter case (simulations $H 4 D 102^{1,2,3}$ of KU2017 - images not shown), regular streamwise-aligned ridges are either 

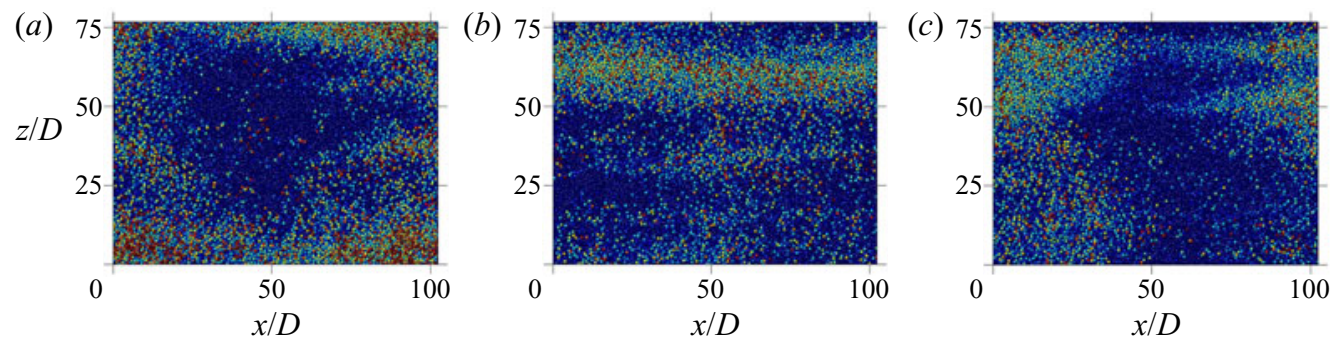

FIGURE 6. Instantaneous snapshots of the sediment bed of case $H 2 D 102^{1}$ at $(a) t \approx 471 T_{b}$, (b) $t \approx 619 T_{b}$ and $(c) t \approx 687 T_{b}$, seen from the top of the channel. Colour scheme is the same as in figure 2 .
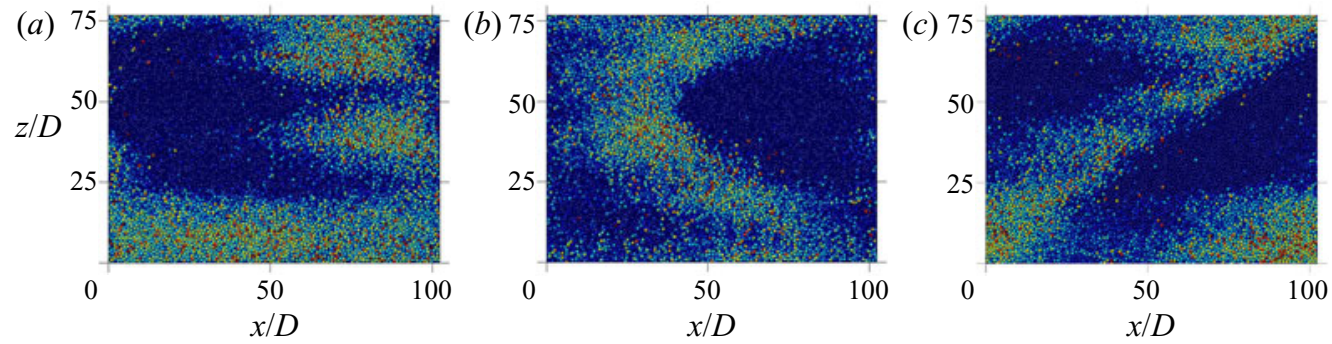

FIGURE 7. Instantaneous snapshots of the sediment bed of case $H 2 D 102^{2}$ at $(a) t \approx 300 T_{b}$, (b) $t \approx 352 T_{b}$ and $(c) t \approx 490 T_{b}$, seen from the top of the channel. Colour scheme is the same as in figure 2.

displaced by the higher, dominating transverse bedforms or do exist superimposed to the former ones.

An important consequence of the observed behaviour in cases $H 2 D 102^{1}$ and $H 2 D 102^{2}$ is that the bed height is clearly two-dimensional. This means that the spanwise-averaged sediment bed and flow field will not correctly describe the physical processes that lead to the formation of these type of bedforms. In the remainder of the current work, we will therefore analyse the sediment bed in its entire streamwise and spanwise dimensions and omit the spanwise averaging (cf. the definition in § 4.2).

Figure 8 shows the time evolution of the root mean square of the two-dimensional sediment bed height fluctuation $\sigma_{h}^{2 D}$. All cases exhibit an exponential growth interval, but with clearly different growth rates, as indicated by the fitted exponential curves presented in figure 8. It is important to keep in mind that in contrast to the spanwise-averaged case, the two-dimensional measure $\sigma_{h}^{2 D}$ takes into account streamwise and spanwise variations of the fluid-bed interface, which means that a change in $\sigma_{h}^{2 D}$ with time can be a sign of evolving ridges or transverse patterns likewise. Indeed, the detailed analysis of a two-dimensional Fourier decomposition of the bed height perturbation which is presented below shows that the amplitude of variations in both directions is of similar order. In order to determine the scaling of the initial growth rate of the sediment bed height fluctuation, however, one would require a larger database and a larger number of individual runs at each parameter point to allow for ensemble averaging similar to case $H 4 D 102^{1,2,3}$. Nevertheless, it would be of great interest to elucidate the influence of the relevant parameters on the initial growth rate of $\sigma_{h}^{2 D}$ such as the Reynolds number $R e_{b}\left(R e_{\tau}\right.$, respectively) and the relative submergence $H_{f} / D$ in a future study. After approximately 300 bulk time units, 


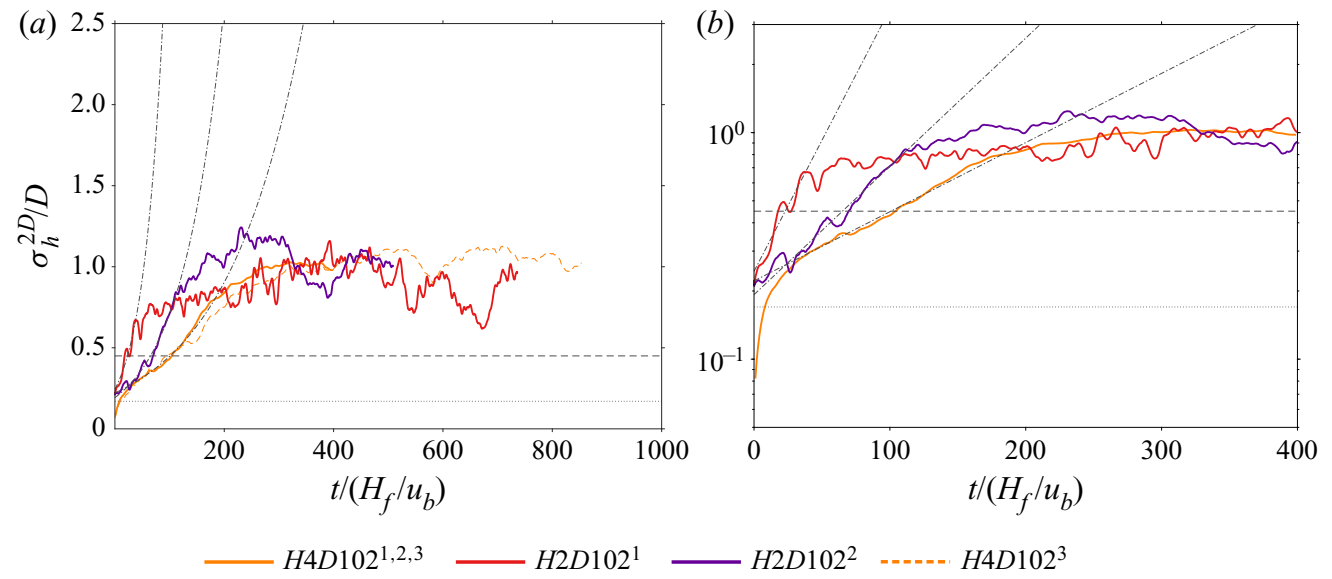

Figure 8. (a) Time evolution of the two-dimensional root mean square of the bedform amplitude in cases $H 4 D 102^{1,2,3}, H 2 D 102^{1}$ and $H 2 D 102^{2}$ normalized by the particle diameter $\sigma_{h}^{2 D} / D$. Time is scaled in bulk time units $T_{b}$. The data of case $H 4 D 102^{1,2,3}$ are presented as an ensemble average over the three simulations. Additionally, the individual evolution of run $H 4 D 102^{3}$ is presented. The dashed-dotted lines represent exponential curves of the form $\sigma_{h} / D=A \exp \left(B t / T_{b}\right)$ which have been found to best fit the evolution of $\sigma_{h}^{2 D}$ during the initial growth period of the respective case. The coefficients are as follows: $H 4 D 102^{1,2,3}: A=0.2215$, $B=0.0070 ; H 2 D 102^{1}: A=0.2441, B=0.0266 ; H 2 D 102^{2}: A=0.1926, B=0.0130$. Note that in case $H 4 D 102^{1,2,3}$, the exponential curve best fits the ensemble average over the three runs. $(b)$ Same data as $(a)$, but represented in semi-logarithmical scale.

all cases eventually reach what could be called an asymptotic state. We observe that the pattern amplitude attains fully developed averaged values of order comparable to one particle diameter for all cases, which suggests that at the given parameter point shown in figure 8 , the averaged value of $\sigma_{h}^{2 D}$ in the final interval does not strongly depend on the value of the mean fluid height $H_{f}$.

The analysis of $\sigma_{h}^{2 D}$ has shown that the sediment bed becomes unstable in all three cases. However, as $\sigma_{h}^{2 D}$ is an integral measure for the bed evolution, it cannot provide further information about the evolution and interaction of single unstable modes. In particular, it does not allow us to determine the role of streamwise and transverse modes separately. For this reason, the evolution of the bed height perturbation will be further analysed in Fourier space for the different streamwise and spanwise wavenumbers. To this end, we compute the single-sided amplitude spectra $\hat{A}_{(k, l)}$ (for the physically relevant non-negative wavenumbers $k, l \geq 0)$ as twice the absolute value of the coefficient $\hat{h}_{b(k, l)}\left(\kappa_{k}^{1}, \kappa_{l}^{3}, t\right)$, which is the discrete Fourier transform of the sediment bed height perturbation in physical space, $h_{b}^{\prime}(x, z, t)$. In the above expression, $\kappa_{k}^{d}$ is the wavenumber of the $k$ th mode in spatial direction $d$ (where $d=1,3$ corresponds to the $x$-and $z$-direction, respectively).

Figure 9 shows the time evolution of the single-sided amplitude spectra for the most dominant modes in case $H 4 D 102^{3}, H 2 D 102^{1}$ and $H 2 D 102^{2}$, respectively. In case $H 4 D 102^{3}$, the wave with modes $(1,0)$ (first harmonic in the streamwise, constant in the spanwise direction) attains the highest amplitudes and thus clearly dominates the spectra. In good agreement with the evolution of $\sigma_{h}^{2 D}$, this mode first increases exponentially during the initial 200-250 bulk time units, before it settles at values which fluctuate somewhat around an asymptotic mean. Note that only the first two harmonics of the pure streamwise 


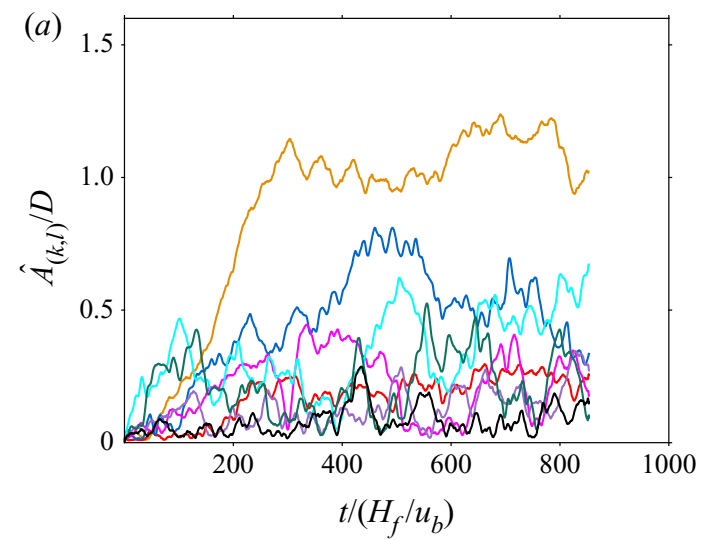

(b)

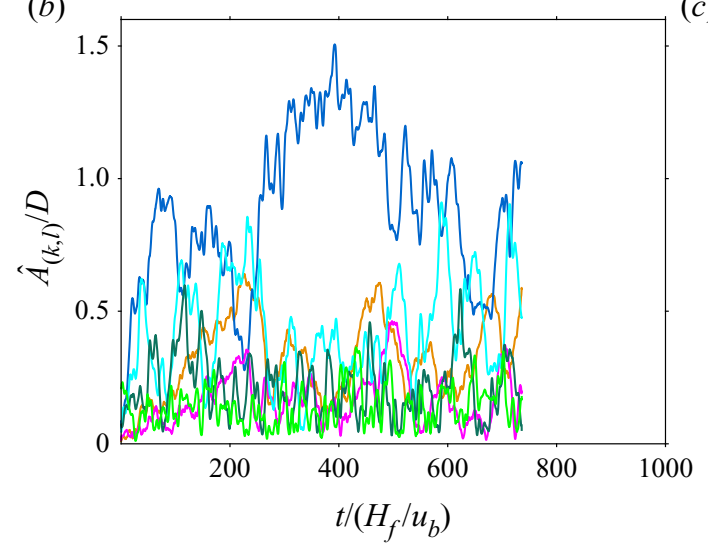

(c)

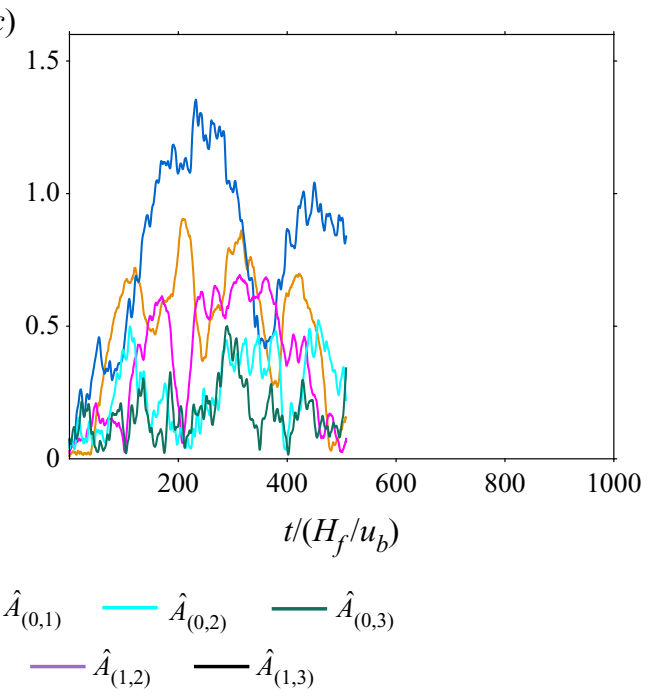

FIGURE 9. Time evolution of the single-sided amplitude spectra for the most dominant modes normalized with the particle diameter $\hat{A}_{(k, l)} / D$ for cases $(a) H 4 D 102^{3}$, (b) $H 2 D 102^{1}$ and (c) $H 2 D 102^{2}$. Note that only those modes are defined as dominant which exceed a value of $\hat{A}_{(k, l)}=0.30 D$ during the simulation. The notation $\hat{A}_{(k, l)}$ indicates that the amplitude corresponds to the mode with streamwise and spanwise wavenumbers $\kappa_{k}^{1}$ and $\kappa_{l}^{3}$, respectively.

waves $\lambda_{(1,0)}$ and $\lambda_{(2,0)}$ exceed a value of $\hat{A}_{(k, l)}=0.30 D$ during the observation interval, highlighting that, in these cases with a comparably short domain, a very sparse range of wavenumbers is amplified, and is then available to form the sediment pattern. On the other hand, waves of the first three pure spanwise harmonics $\lambda_{(0,1 \ldots 3)}$ are found to be significant. In the first approximately 100-150 bulk time units, these pure spanwise modes dominate the amplitude spectra, which reflects the formation of initial streamwise aligned ridges that has been observed by KU2017. In the subsequent quasi-steady phase of bedform evolution, pure spanwise modes are still present with finite but smaller amplitudes compared to $\hat{A}_{(1,0)}$, reaching maximum values $\hat{A}_{(0,1)}=0.80 D$. This is in good agreement with the observation of the aforementioned authors, that streamwise elongated patterns are still visible on the upstream face of the transverse bedforms, once these latter have reached a quasi-steady state. In addition, several diagonal waves including modes 
larger than zero in both directions evolve, but they do not reach amplitudes higher than $\hat{A}=0.5 D$. The time evolution of the amplitude spectra in the new simulations $H 2 D 102^{1}$ and $H 2 D 102^{2}$ (cf. figure $9 b, c$, respectively), on the other hand, is dominated by the first pure spanwise oscillating wave mode $(0,1)$ during almost the whole observation interval, attaining maximum values of $\hat{A}_{(0,1)} / D$ above unity. In comparison, the amplitude of the first pure streamwise oscillating wave mode $(1,0)$ which represents in these cases the only significant pure streamwise wave attains somewhat smaller maximum values with $\hat{A}_{(1,0)} / D$ not exceeding unity. In general, the amplitude of the single modes exhibit higher fluctuations than those in case $H 4 D 102^{3}$, leading to differences of approximately $1 D$ between the highest and the lowest attained values of a single mode. During the entire simulation, none of the modes reach a plateau regime which would indicate a quasi-steady state of the bed. These findings match our observations, that both systems $H 2 D 102^{1}$ and $H 2 D 102^{2}$ show a sequence of different alternating bedform configurations composed of a number of streamwise and spanwise unstable modes without eventually reaching a quasi-steady state. In particular, transverse sediment bed waves evolve and, even though they are observed to be of smaller amplitude than their spanwise directed counterparts, they are involved in the formation of three dimensional patterns. Eventually, this reveals that, indeed, a sediment bed can become unstable for a domain length $L_{x} / H_{f}<3$, indicating that the lower bound for the most unstable wavelength as reported by KU2017 does not scale with the mean fluid height $H_{f}$. The three-dimensional pattern evolution, however, requires further investigation.

\section{Discussion}

Our observations in the previous section suggest that in the investigated parameter range, the minimal unstable wavelength depends on the particle diameter, whereas it seems to be rather unaffected by variations of the mean fluid height. In the following, we will compare our results with measurements as well as proposed scaling relations from experimental and theoretical studies.

In figure 10, we present an overview of initial mean pattern wavelengths from our current and previous direct numerical simulation studies together with values determined in laboratory experiments. The observed wavelengths are shown as functions of the particle diameter $D$ and of the mean fluid height $H_{f}$, respectively. Note that for stable sediment beds (indicated by open symbols in figure 10), the definition of an initial pattern wavelength is meaningless. Here, we give instead the streamwise domain length $L_{x}$, which indicates the maximum possible wavelength that did not evolve during the simulation. In cases $H 2 D 102^{1}$ and $H 2 D 102^{2}$, on the other hand, we have observed the sediment bed to evolve both in the streamwise and spanwise direction. In these two cases we equally associate the value of the domain length $L_{x}$ with the most unstable wavelength, since the study of the single-sided amplitude spectra of the sediment bed height fluctuation shows that the most dominant streamwise mode is the one with indices $(1,0)$, i.e. the first streamwise harmonic which is constant in the spanwise direction. It should be further mentioned that with cases $H 2 D 052$ and $H 4 D 052$, two additional new cases have been added to the parameter plane, which were not part of the analysis in the previous section (cf. tables 1 and 2 for the physical and numerical parameters). In both cases the bed is observed to remain stable, i.e. no transverse patterns form during the simulation. The experimental data shown in figure 10 stem from measurements in channel flow by Langlois \& Valance (2007) as well as in closed-conduit flows by Coleman et al. (2003) and Cardona Florez \& Franklin (2016). 


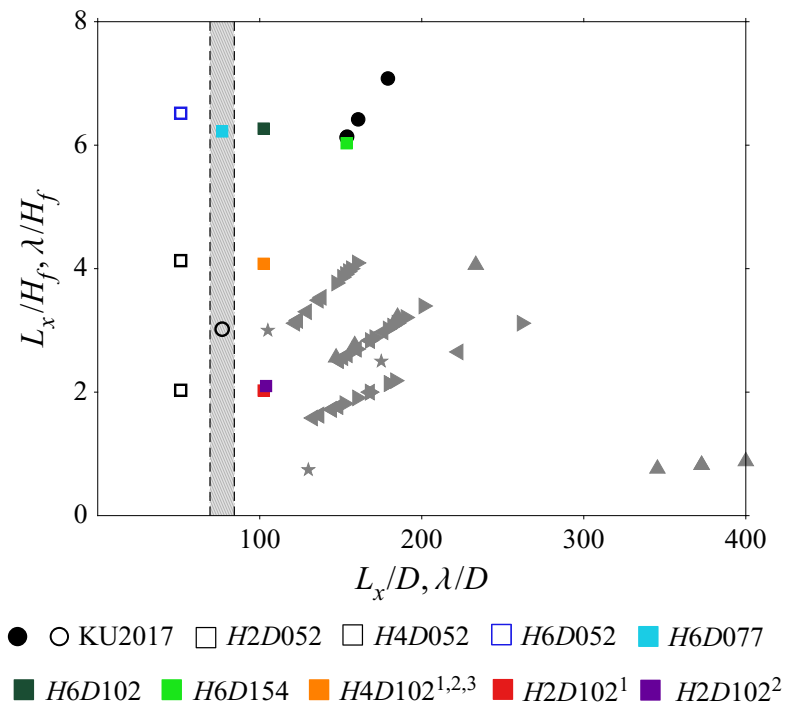

FIGURE 10. Minimal unstable wavelengths in numerical simulations and experiments as functions of the particle diameter $D$ and the mean fluid height $H_{f}$. Filled symbols indicate unstable sediment beds, whereas open symbols are used for stable systems. The presented wavelengths are determined as the time average of the mean wavelength $\lambda_{h}$ over the final time period $T_{o b s}^{s}$. It should be noted that, for stable systems as well as for cases $H 2 D 102^{1}$ and $H 2 D 102^{2}$, in which three-dimensional patterns evolve, the streamwise box length is given instead of the spanwise-averaged mean wavelength (cf. the detailed discussion in the text). Wavelengths measured in experiments are presented with the following grey symbols: Coleman et al. (2003) (closed-conduit, A), Langlois \& Valance (2007) (channel, «), Cardona Florez \& Franklin (2016) (closed-conduit, $<$ for the first and $>$ for the last measured wavelength). Note that in the experimental studies, no free surface is present. Thus, the experimentally determined wavelengths are normalized with the half mean fluid height. Based on the results of our analysis, we expect the minimal unstable wavelength in the vertical grey region around $\lambda / D=80$.

In our direct numerical simulations, we have observed transverse pattern formation only in cases with a relative streamwise domain length $L_{x} / D \geq 76.8$, while the sediment bed remained stable for all simulations below this limit, irrespective of the fluid height. At a domain length $L_{x} / D=76.8$, we have observed case $H 6 D 077$ to become unstable, but the shape and asymmetry of the evolving pattern is seen to substantially deviate from the observed values in sufficiently long domains such as the cases of KU2017 with a box length of $L_{x} / D=O\left(10^{3}\right)$. A possible reason for this observation is that in $H 6 D 077$, the system can only choose between 8 harmonics with a wavelength higher than $O(D)$. The interaction of this small number of discrete modes may not lead to the same interface that would form in a sufficiently long domain. Interestingly, KU2017 report a case with the same relative domain length $L_{x} / D=76.8$ in which the sediment bed remained flat. We therefore expect that a box length $L_{x} / D=76.8$ is in the vicinity of the sought threshold wavelength, such that at this parameter point, already small differences in the configuration might tip the system in either way. If the domain length is even further decreased (as in cases $H 2 D 052, H 4 D 052$ and H6D052) the bed remains macroscopically flat.

In the considered experimental studies, the shortest measured wavelength $\lambda / D \approx$ 105 is clearly above the critical wavelength determined in the present work. In our 
simulations, the shortest box which allowed a single bedform to reach an asymptotic state with an asymmetric triangular shape was observed for a very similar box length $L_{x} / D=102.4$. Nevertheless, the aspect ratio is still smaller than in the longer cases $H 6$, $H 7$ and $H 12$ of KU2017, which might be an indication that the patterns did not yet reach their final state and would further evolve if their growth was not hindered by the limited domain size. Indeed, the majority of the experimental data points concentrate in a range of $\lambda / D=120-250\left(\lambda / H_{f}=0.5-4.0\right)$. In good agreement, all numerical simulations with $150 \leq L_{x} / D \leq 300$ develop patterns with a mean wavelength in the range $150 \leq \lambda / D \leq 180$ and a self-similar profile. These observations further support the suggestion of KU2017, that the bedforms finally settle at a wavelength of approximately $150 \leq \lambda / D \leq 180$. Similarly, Coleman \& Nikora $(2009,2011)$ report a lower bound for the preferred initial wavelength of $\lambda / D=130$. Eventually, the occurrence of patterns with a wavelength $\lambda / H_{f}<3$ in numerical simulations and experiments which can be seen in figure 10 excludes a possible scaling of the threshold with the mean fluid height which KU2017 could not exclude based on their limited data. This observation is further supported by the results of the analysis in the preceding section: cases with identical relative streamwise domain length $L_{x} / D$ but varying $L_{x} / H_{f}$ ratio attain similar final values for all studied geometrical parameters and spanwise-averaged bedform profiles.

Compared to the wavelengths observed in experiments, the initial wavelengths predicted by most linear stability analyses are smaller by at least one order of magnitude (Langlois \& Valance 2007; Ouriemi et al. 2009). However, in some recent studies, the prediction of the most unstable wavelength could be improved taking additional physical effects into account. For instance, initial unstable pattern wavelengths similar to those determined in experiments were found after introducing an additional stabilizing effect in form of a phase lag between the boundary shear stress and the particle flow rate into the linear stability analyses by several authors (Charru 2006; Charru \& Hinch 2006; Fourriere et al. 2010). This phase shift, which is usually termed as characteristic saturation length $L_{\text {sat }}$, has been reported to be a function of the particle diameter, attaining values of the order of 10 times the particle diameter for pure bedload transport (Claudin, Charru \& Andreotti 2011). For the special case of sand grains, Fourriere et al. (2010) present saturation lengths between 7 and 15 grain diameters. In their subsequent stability analysis, these authors predict for sufficiently large fluid heights most amplified wavelengths in a range of $\lambda / L_{s a t}=15-20$, which is equivalent to a range $\lambda / D=105-300$, when assuming that $L_{\text {sat }}=7-15$. This range of most amplified wavelengths overlaps with the values found in simulations and the mentioned experimental studies.

The findings of Colombini \& Stocchino (2011), on the other hand, differ from our observations and the results of Fourriere et al. (2010). For the range of Galileo numbers considered in the present study, the authors report only one unstable region that depends on the fluid height exclusively. In contrast, unstable wavelengths scaling with the particle diameter, which could be related to a ripple instability, appear in their model for $G a \leq 14$ only. It should be, however, noted that the model of Colombini \& Stocchino (2011) strongly depends on the roughness height and, consequently, on the relative submergence $H_{f} / D$, which is in their case at least one order of magnitude higher than in our numerical simulations. Therefore, a direct comparison of the predicted critical wavelengths with our results is not possible.

Finally, let us turn to the question of the significance of the confirmed scaling of the wavelength of initial ripple patterns. As previously argued by Coleman \& Nikora (2009) we believe that these patterns scale with the grain diameter as a consequence of the wake effect of bed perturbations in the initial stages (i.e. small lumps of dislodged particles). 
Since initial seeds for pattern formation are composed of bunches of those particles which are being dislodged, these initial seeds invariably have vertical extents of $O(D)$. Due to their frequent contact with the bed, the seeds travel at a smaller speed than the surrounding fluid, and a wake forms in the region downstream of the seed crest. As a consequence, the probability that a particle will be dislodged and set into motion decreases in the wake of the seed, which in turn means that a second seed being located within some effective distance downstream of a given seed will receive less sediment supply from upstream, and it will therefore grow at a smaller rate or it will not grow at all. This will lead to initial pattern wavelengths which will be larger than the effective wake length of the incipient seeds. Analogously, in our numerical experiments with constrained streamwise domain lengths this effect suppresses all pattern formation if the period is below the effective wake length. This scenario applies to the bedload dominated regime; in the case of dominantly suspended load, other length scales are expected to come into play, such as the deposition length (Claudin et al. 2011).

In order to obtain a first estimate of the effective wake length of an initial seed let us perform the following idealization. Consider a single sphere in uniform, steady inflow with approach velocity $u_{\infty}$. Classical analysis for laminar wakes (Schlichting 1979; Wu \& Faeth 1993) shows that the streamwise velocity deficit on the axis behind the sphere varies as the inverse of the downstream distance $x$ in the self-similar regime, such that we can write for the distance it takes the wake to recover up to $T_{u} u_{\infty}$ :

$$
\left(x-x_{0}\right) / D=R e_{D} C_{D} /\left(32 T_{u}\right) \approx\left(0.17 R e_{D}^{0.6}+0.66\right) / T_{u},
$$

where $x_{0}$ is the virtual origin, $R e_{D}=D u_{\infty} / v$ the sphere Reynolds number, $C_{D}$ the drag coefficient for which in the second step we have substituted a standard drag formula (Clift, Grace \& Weber 1978, p. 112, table 5.2), and fitted the result and $0<T_{u}<1$. For a threshold of a few per cent $\left(T_{u}=0.05\right.$, say) this estimate yields a wake length of $O(10)$ particle diameters over a range of Reynolds numbers of interest. The isolated sphere wake is indeed a drastic simplification of the actual sediment pattern seed. First, the incoming flow is not quiescent but turbulent: it is well known that a laminar wake in a turbulent background flow tends to recover somewhat faster than its counterpart in laminar flow (Amoura et al. 2010; Zeng, Balachandar \& Najjar 2010). Second, the sphere is not isolated, but it is located near a complex-shaped, evolving sediment bed: some researchers have indeed considered this problem (for a fixed single sphere), but the scaling of the wake length with Reynolds number was not explicitly obtained (Dey et al. 2011; Lee \& Balachandar 2017). In order to gauge the latter influence, we have performed a simulation with a single spanwise row of spheres placed adjacent to a macroscopically flat sediment bed holding all particles fixed. In this case the flow is laminar with a Reynolds number $R e_{b}=300$, and the particle diameter in viscous units measures $D^{+}=3.5$ (with $H_{f} / D=8$ ), while the particle Reynolds number computed with the undisturbed flow velocity at the wall distance of the tops of the perturbation particles measures $R e_{D}=$ $D u_{f}\left(y_{p}+D / 2\right) / v=17$. The effective wake length (assuming $\left.T_{u}=0.05\right)$ is approximately equal to $50 D$ in this case, confirming the above order-of-magnitude estimate. Note that we have also confirmed that under laminar flow conditions at $R_{b}=300$ a consistent threshold for the pattern wavelength can be obtained through our box-size constraining approach, analogously to the turbulent flow cases discussed up to now (cf. appendix B for details).

Despite the simplicity of the wake-effect argument in its current form, it suggests that the lower threshold of the initial pattern wavelength when scaled in particle diameters should exhibit a Reynolds number dependence. Although not extremely large, the influence of the particle Reynolds number (such as suggested by (6.1)) should be detectable 
with carefully designed experiments and/or numerical simulations. This constitutes a worthwhile research question for a future study.

\section{Conclusion}

In the present study, we have investigated the initial stage of subaqueous pattern formation in a turbulent open channel flow by means of direct numerical simulations with fully resolved particles. The main contribution is to address the question of scaling of the initial bedform wavelength with either the particle diameter $D$ or with the main fluid height $H_{f}$. Both scaling relations have been proposed by different authors in the past decades, but up to the present day, there is no clear consensus about the correct scaling length. In a recent paper, Kidanemariam \& Uhlmann (2017) have observed a lower bound for the most unstable wavelength $\lambda_{\text {th }}$ in a range $(75-100) D$ (equivalent to (3-4) $H_{f}$, respectively). However, it was not possible in that study to further tackle the problem of the correct scaling, since the relative submergence $H_{f} / D$ was constant in all of their simulations. In the present work, we have therefore performed and analysed two sets of simulations, in which we have varied the relative streamwise box lengths $L_{x} / D$ and $L_{x} / H_{f}$ independently, which allowed us to investigate the influence of both length scales on the initial pattern wavelength exclusively. Consequently, the relative submergence has been varied in the range $H_{f} / D=7.86-50.59$.

Our findings imply a scaling of the initial wavelength with the particle diameter, while it seems to be rather unaffected by variations of the mean fluid height. In particular, a lower bound for the most unstable wavelength has been found around a streamwise domain length of approximately $L_{x} / D=80$. In cases with a shorter relative box length $L_{x} / D$, transverse pattern formation was effectively suppressed, indicating that the domain length is not sufficient to accommodate the minimal unstable wavelength. However, the observed pattern with a wavelength close to the proposed threshold exhibits remarkable differences compared to patterns in sufficiently long domains (cf. for instance the cases with $L_{x} / D=O\left(10^{3}\right)$ of Kidanemariam \& Uhlmann 2017), i.e. in very marginal boxes it does not reach an asymptotic state during the observation time and shows a more symmetric spanwise-averaged profile than in the longer cases.

The predicted range of initial pattern wavelengths is in good agreement with values measured in laboratory channel and closed-conduit experiments, which concentrate predominantly in a range $\lambda / D=120-250$. Furthermore, good agreement is also observed with wavelengths predicted as $\lambda / D=105-300$ in the stability analysis of Fourriere et al. (2010). Note that, in their study, the dependence of the most amplified wavelength on the particle diameter appears indirectly in form of a scaling with a saturation length $L_{s a t}$, which in turn is a function of the particle diameter.

We have argued that the observed dependence of the minimal unstable wavelength of sediment patterns upon the particle diameter is essentially a consequence of the effective wake length of the initial seeds, as already put forth by Coleman \& Nikora (2009). Both, scaling arguments based on laminar wakes of isolated spheres as well as numerical estimates of the wake effect of small-amplitude perturbations in laminar open channel flow support this scenario. It was also argued that the wake effect should exhibit a (mild) Reynolds number dependence which has so far not been clearly demonstrated. Therefore, further work is required in this direction. One possibility would be to identify initial seeds (during the early stages of pattern development) in either experimental or numerical realizations, and to quantify the wake effect statistically, either indirectly through the velocity field, or directly through conditional averaging of the particle flux. 
Another remarkable phenomenon has been observed in the cases with the highest relative submergence $H_{f} / D \approx 50$ in the form of streamwise and spanwise sediment features, that are seen to either concur or interact with each other, allowing for the formation of three-dimensional patterns. Our analysis of the single-sided amplitude spectra of the sediment bed height perturbation has shown that the evolution of the sediment bed is dominated by several streamwise and spanwise oriented waves of comparable amplitude. In the remaining simulations, streamwise aligned patterns appeared, if at all, mainly as a predecessor of the transverse patterns in the first few bulk time units of the simulations, but at clearly lower amplitude than their transverse oriented counterparts. Since the focus of the current study was on the scaling of transverse patterns, it was out of the scope to investigate in detail the reasons for this different sediment bed evolution. However, it would be of great interest to determine the parameters that are responsible for the amplification of these spanwise waves. In this context, it should be investigated in future studies how the initial subaqueous pattern formation is affected by constraints on the spanwise length scales.

\section{Acknowledgements}

The current work was supported by the German Research Foundation (DFG) through grants UH242/2-1 and UH242/12-1. Part of the work was performed on the supercomputer ForHLR II at the Steinbuch Centre for Computing funded by the Ministry of Science, Research and the Arts Baden-Württemberg and by the Federal Ministry of Education and Research. The remaining simulations have been carried out on SuperMUC at the Leibniz Supercomputing Centre at the Bavarian Academy of Science and Humanities. Disk space for presenting supplementary material online is provided by the 4TU.Centre for Research Data at the TU Delft. The computer resources, technical expertise and assistance provided by the staff at these computing centres are gratefully acknowledged. We thank M. Krayer for his support in defining the fluid-bed interface.

\section{Declaration of interests}

The authors report no conflict of interest.

\section{Supplementary movies}

Supplementary movies are available at https://dx.doi.org/10.4121/uuid:7eb6a0be-ff834883-9d99-31 daaa6a2863.

\section{Appendix A. Sensitivity to the choice of the Coulomb friction coefficient}

As mentioned in $\S 2$, two of our performed simulations feature a slightly higher Coulomb friction coefficient $\mu_{c}=0.5$ compared to the remaining cases, where a value of $\mu_{c}=0.4$ was chosen. In the following, we show that the difference in this parameter has a minor effect on the eventually developed pattern and, in particular, that it does not affect the stability or instability of the sediment bed. To this end, we have recomputed case $H 6 D 102$ keeping all parameters except for the Coulomb friction coefficient, which we reduced to the value $\mu_{c}=0.4$ as in the remaining simulations. Furthermore, we performed another simulation with identical parameters and $\mu_{c}=0.4$, but with a different initial flow field in order to check for the sensitivity to the initial data.

In figure 11, we compare the time evolution of the root mean square sediment bed height fluctuation $\sigma_{h}$ for the three simulations. It shows clearly that the bed becomes unstable 

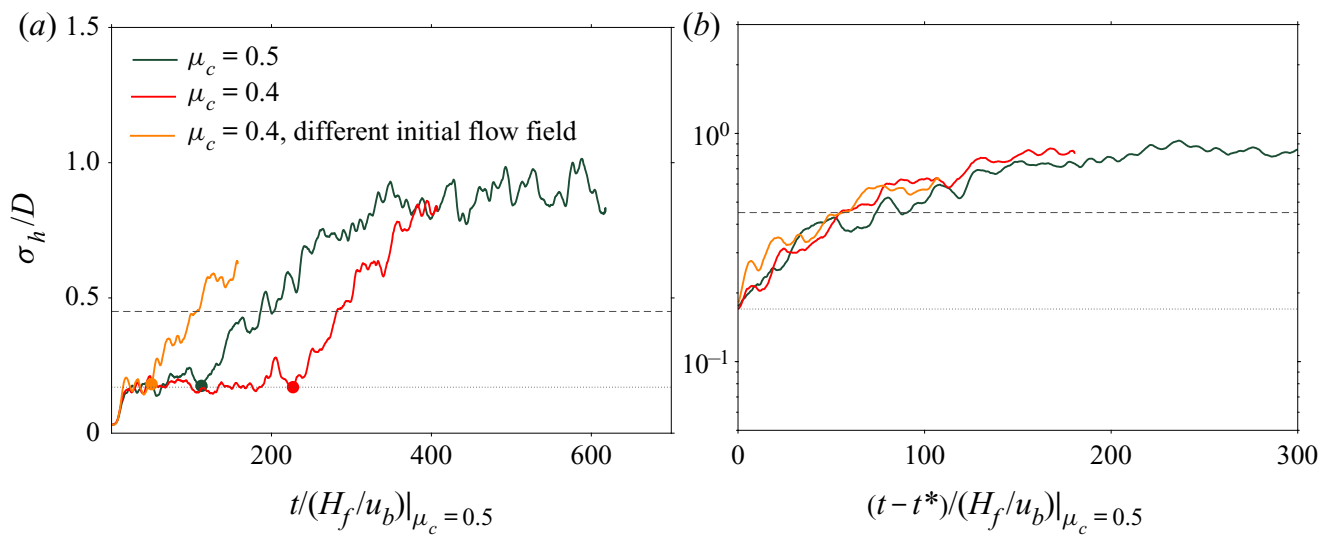

FIGURE 11. (a) Time evolution of the root mean square of the bedform amplitude normalized by the particle diameter $\sigma_{h} / D$ for case $H 6 D 102$ using different Coulomb friction coefficients. (b) Same as in (a), but in semi-logarithmic scale. The origin is shifted to the instant in time at the onset of the macroscopic growth period $t^{*}$, which is indicated by filled circles in $(a)$.

\begin{tabular}{ccccccccc}
\hline Case & $\operatorname{Re}_{b}$ & $R e_{\tau}$ & $\rho_{p} / \rho_{f}$ & $G a$ & $D^{+}$ & $H_{f} / D$ & $H_{b} / D$ & $\theta$ \\
$-H 6 D 052_{\text {lam }}$ & 303 & 29.84 & 2.5 & 8.95 & 3.62 & 8.24 & 4.56 & 0.16 \\
$-H 12 D 102_{\text {lam }}$ & 303 & 30.12 & 2.5 & 8.95 & 3.65 & 8.25 & 4.55 & 0.17
\end{tabular}

TABle 3. Physical parameters of the laminar simulations.

$\begin{array}{cccccccc}\text { Case } & {\left[L_{x} \times L_{y} \times L_{z}\right] / D} & L_{x} / H_{f} & D / \Delta x & \Delta x^{+} & N_{p} & T_{o b s} / T_{b} & T_{o b s}^{s} / T_{b} \\ -H 6 D 052_{\text {lam }} & 51.2 \times 12.8 \times 25.6 & 6.22 & 10 & 0.36 & 5086 & 780 & 671 \\ -H 12 D 102_{\text {lam }} & 102.4 \times 12.8 \times 25.6 & 12.43 & 10 & 0.37 & 11482 & 1096 & 988\end{array}$

TABLE 4. Numerical parameters of the laminar simulations.

for both values of $\mu_{c}$ and that, furthermore, the growth rate is very similar in all three simulations, although the instant in time of the onset of the macroscopic growth regime differs. These observations indicate that the bedform evolution is not sensitive to a slight increase of the chosen $\mu_{c}$ in the observed range. Therefore, we will not further differentiate between the cases with $\mu_{c}=0.4$ and $\mu_{c}=0.5$ in the remainder of this study.

\section{Appendix B. Additional simulations of a sediment bed under laminar flow conditions}

This section describes additional simulations in the laminar regime, with otherwise the same set-up as in the main part of the manuscript. The first of these new cases, which will henceforth be denoted as case $H 6 D 052_{\text {lam }}$, is identical to case $H 6 D 052$ (cf. table 1) concerning the initial state of the particle bed and the box dimensions, but the bulk Reynolds number in this new case has been reduced by a factor of 10 compared to the turbulent baseline case. The Galileo number has been adapted accordingly in order 

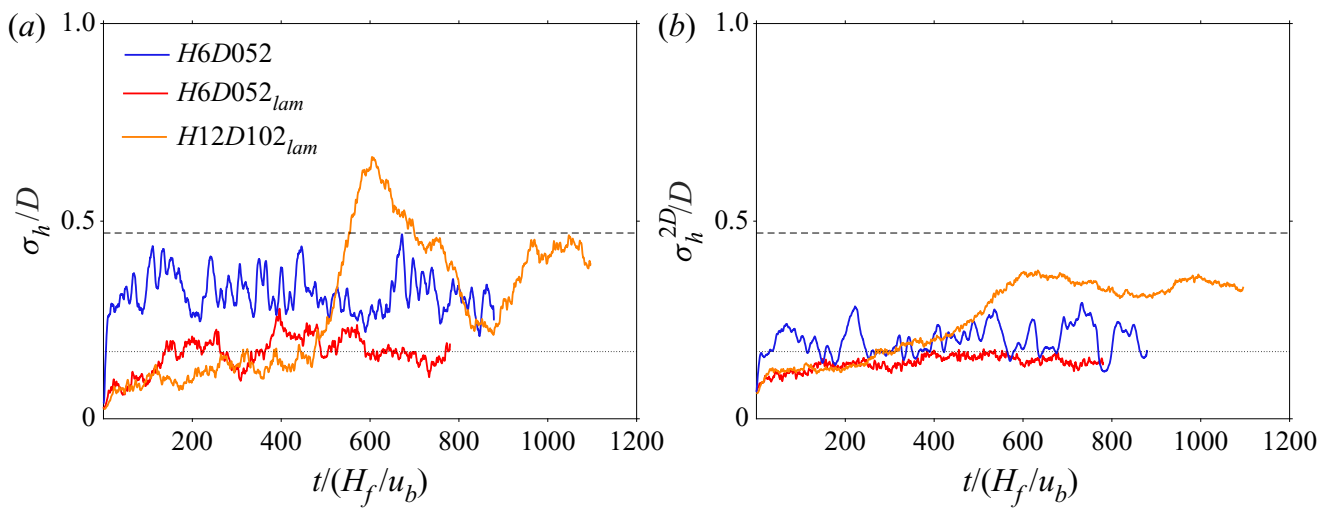

FIGURE 12. Time evolution of the $(a)$ one-dimensional and $(b)$ two-dimensional root mean square of the bedform amplitude normalized by the particle diameter for cases $H 6 D 052$, $H 6 D 052_{\text {lam }}$ and H12D102 lam .
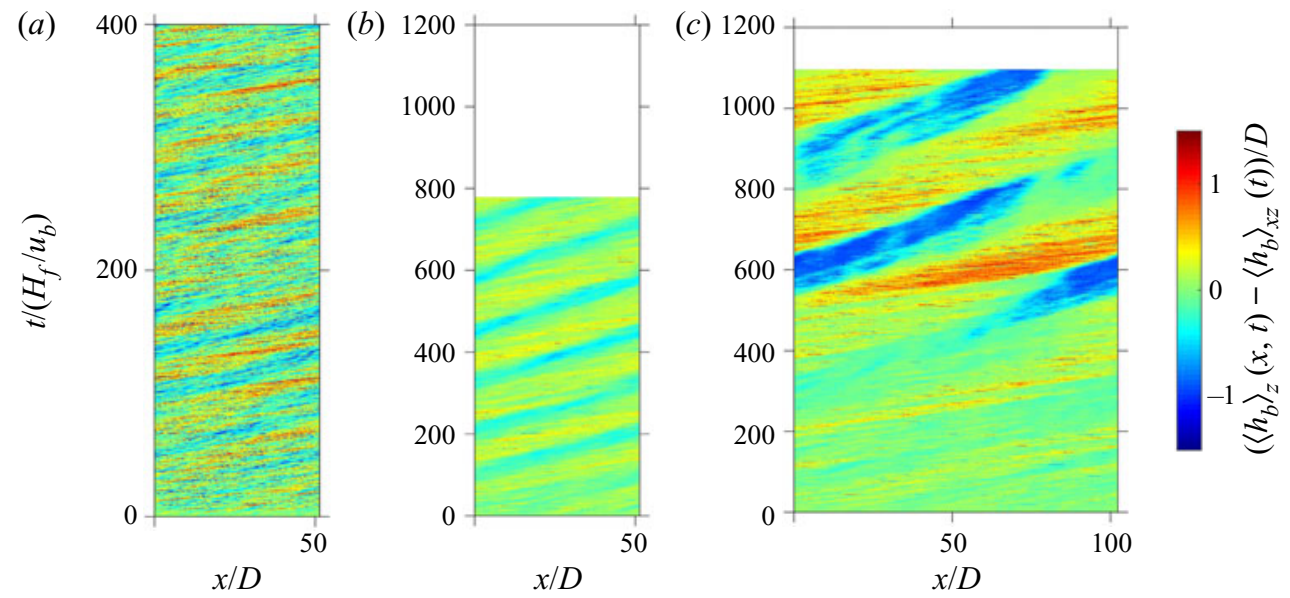

FIGURE 13. Space-time evolution of the fluctuation of the spanwise-averaged fluid-bed interface in multiples of the particle diameter for cases (a) H6D052, (b) H6D052lam and (c) H12D102 lam. Note that the range of values shown on the ordinate in the turbulent case (panel $a$ ) differs from that of the laminar cases (panels $b, c$ ).

to reach a comparable Shields number as in the turbulent cases. For a second simulation $H 12 D 102_{\text {lam }}$, the streamwise extent of the computational domain of case $H 6 D 052_{\text {lam }}$ has been doubled, i.e. $L_{x} / D=102.4$, while the remaining physical and numerical parameters are identical to those in case $H 6 D 052_{\text {lam }}$. An overview over all relevant physical and numerical parameters in the two simulations is provided in tables 3 and 4, respectively. Based on the critical domain length observed in the turbulent regime, one would expect the sediment bed in case $H 6 D 052_{\text {lam }}$ to remain essentially flat without allowing a transverse sediment pattern to grow, while in $H 12 D 102_{\text {lam }}$, the box length should be sufficiently long to accommodate a single streamwise oscillating sediment wave. Figure 12 shows the amplitude of the bed height variation, corresponding to figures 3 and 8 of $\S 5$. In particular, from the amplitude of the two-dimensional sediment bed extraction (figure 12b) it can be seen that the elongated domain allows for the formation of significant patterns, albeit at a 
much smaller rate than the turbulent counterparts in figure 8. Conversely, the bed in the shorter domain $H 6 D 052_{\text {lam }}$ indeed remains featureless, as was already observed for the turbulent case $H 6 D 052$.

Figure 13 shows a complementary view of the growth process, by providing space-time plots of the spanwise-averaged bed elevation for the three cases included in figure 12. Here, it becomes evident that seeds of patterns are distinguishable in all three cases, but only do they grow to significant amplitudes in the sufficiently long domain. The main distinction between the laminar and turbulent regime is the substantially lower propagation speed of the seeds in the former.

\section{REFERENCES}

Amoura, Z., Roig, V., Risso, F. \& Billet, A.-M. 2010 Attenuation of the wake of a sphere in an intense incident turbulence with large length scales. Phys. Fluids 22, 055105.

Andreotti, B. \& Claudin, P. 2013 Aeolian and subaqueous bedforms in shear flows. Phil. Trans. R. Soc. A 371 (2004), 20120364.

Bagnold, R. A. 1956 The flow of cohesionless grains in fluids. Phil. Trans. R. Soc. Lond. A 249 (964), 235-297.

BEST, J. 2005 The fluid dynamics of river dunes: a review and some future research directions. J. Geophys. Res. 110, F04S02.

Cardona Florez, J. E. \& Franklin, E. D. M. 2016 The formation and migration of sand ripples in closed conduits: experiments with turbulent water flows. Exp. Therm. Fluid Sci. 71, 95-102.

Charru, F. 2006 Selection of the ripple length on a granular bed sheared by a liquid flow. Phys. Fluids 18 (12), 121508.

Charru, F., Andreotti, B. \& Claudin, P. 2013 Sand ripples and dunes. Annu. Rev. Fluid Mech. 45, 469-493.

Charru, F. \& Hinch, E. J. 2006 Ripple formation on a particle bed sheared by a viscous liquid. Part 1. Steady flow. J. Fluid Mech. 550, 111-121.

Charru, F. \& Mouilleron-Arnould, H. 2002 Instability of a bed of particles sheared by a viscous flow. J. Fluid Mech. 452, 303-323.

Claudin, P., Charru, F. \& Andreotti, B. 2011 Transport relaxation time and length scales in turbulent suspensions. J. Fluid Mech. 671, 491-506.

Clift, R., Grace, J. R. \& Weber, M. E. 1978 Bubbles, Drops and Particles. Academic.

Coleman, S. E. \& Eling, B. 2000 Sand wavelets in laminar open-channel flows. J. Hydraul. Res. 38 (5), 331-338.

Coleman, S. E., Fedele, J. J. \& Garcia, M. H. 2003 Closed-conduit bed-form initiation and development. J. Hydraul. Engng ASCE 129 (12), 956-965.

Coleman, S. E. \& Melville, B. W. 1996 Initiation of bed forms on a flat sand bed. J. Hydraul. Engng ASCE 122 (6), 301-310.

Coleman, S. E. \& Nikora, V. I. 2009 Bed and flow dynamics leading to sediment-wave initiation. Water Resour. Res. 45, W04402.

Coleman, S. E. \& NikORA, V. I. 2011 Fluvial dunes: initiation, characterization, flow structure. Earth Surf. Process. Landf. 36 (1), 39-57.

Colombini, M. 2004 Revisiting the linear theory of sand dune formation. J. Fluid Mech. 502, 1-16.

Colombini, M. \& Stocchino, A. 2008 Finite-amplitude river dunes. J. Fluid Mech. 611, 283-306.

Colombini, M. \& Stocchino, A. 2011 Ripple and dune formation in rivers. J. Fluid Mech. 673, $121-131$.

Derksen, J. J. 2015 Simulations of granular bed erosion due to a mildly turbulent shear flow. J. Hydraul. Res. 53 (5), 622-632.

Dey, S., Sarkar, S., Bose, S. K., Tait, S. \& Castro-Orgaz, O. 2011 Wall-wake flows downstream of a sphere placed on a plane rough wall. J. Hydraul. Engng ASCE 137 (10), 1173-1189. 
Edelsbrunner, H. \& MÜCKe, E. P. 1994 Three-dimensional alpha shapes. ACM Trans. Graph. 13 (1), 43-72.

Engelund, F. \& Fredsoe, J. 1982 Sediment ripples and dunes. Annu. Rev. Fluid Mech. 14 (1), 13-37.

Fourriere, A., Claudin, P. \& Andreotti, B. 2010 Bedforms in a turbulent stream: formation of ripples by primary linear instability and of dunes by nonlinear pattern coarsening. J. Fluid Mech. 649, 287-328.

Franklin, E. D. M. 2008 Dynamique de dunes isolées dans un écoulement cisaillé. PhD thesis, Université Toulouse III-Paul Sabatier.

Franklin, E. D. M. \& CharRU, F. 2011 Subaqueous barchan dunes in turbulent shear flow. Part 1. Dune motion. J. Fluid Mech. 675, 199-222.

INGLIS, SIR C. C. 1949 The behaviour and control of rivers and Canals (Part II). Central Waterpower Irrigation and Navigation Research Station, Poona, India.

JiMÉNEZ, J. \& MoIN, P. 1991 The minimal flow unit in near-wall turbulence. J. Fluid Mech. 225, 213-240.

KENNEDY, J. F. 1963 The mechanics of dunes and antidunes in erodible-bed channels. J. Fluid Mech. 16 (4), 521-544.

KenNedY, J. F. 1969 The formation of sediment ripples, dunes, and antidunes. Annu. Rev. Fluid Mech. 1 (1), 147-168.

Kidanemariam, A. G. \& Uhlmann, M. 2014a Direct numerical simulation of pattern formation in subaqueous sediment. J. Fluid Mech. 750, R2.

KidAnemariam, A. G. \& Uhlmann, M. $2014 b$ Interface-resolved direct numerical simulation of the erosion of a sediment bed sheared by laminar channel flow. Int. J. Multiphase Flow 67, 174-188.

KidANEMARIAM, A. G. \& UhlMANN, M. 2017 Formation of sediment patterns in channel flow: minimal unstable systems and their temporal evolution. J. Fluid Mech. 818, 716-743.

LANGlOIS, V. \& VALANCE, A. 2007 Initiation and evolution of current ripples on a flat sand bed under turbulent water flow. Eur. Phys. J. E 22 (3), 201-208.

LAPotre, M. G. A., LAMB, M. P. \& MCElroy, B. 2017 What sets the size of current ripples? Geology 45 (3), 243-246.

LEE, H. \& BALACHANDAR, S. 2017 Effects of wall roughness on drag and lift forces of a particle at finite reynolds number. Int. J. Multiphase Flow 88, 116-132.

Mazzuoli, M., KidAnemariam, A. G. \& UhLmann, M. 2019 Direct numerical simulations of ripples in an oscillatory flow. J. Fluid Mech. 863, 572-600.

Ouriemi, M., Aussillous, P. \& Guazzelli, É. 2009 Sediment dynamics. Part 2. Dune formation in pipe flow. J. Fluid Mech. 636, 321-336.

RAUDKIVI, A. J. 1997 Ripples on stream bed. J. Hydraul. Engng ASCE 123 (1), 58-64.

RichARDS, K. J. 1980 The formation of ripples and dunes on an erodible bed. J. Fluid Mech. 99 (3), 597-618.

VAN RIJN, L. C. 1984 Sediment transport, part I: Bed load transport. J. Hydraul. Engng ASCE 110, (F01009), 1431-1456.

Schlichting, H. 1979 Boundary-Layer Theory, 7th edn. McGraw-Hill.

Seminara, G. 2010 Fluvial sedimentary patterns. Annu. Rev. Fluid Mech. 42, 43-66.

SOULSBY, R. L. \& WhiteHOUSE, R. J. S. 1997 Threshold of sediment motion in coastal environments. In Pacific Coasts and Ports' 97: Proceedings of the 13th Australasian Coastal and Ocean Engineering Conference and the 6th Australasian Port and Harbour Conference, vol. 1, p. 145. Centre for Advanced Engineering, University of Canterbury.

Sumer, B. M. \& BAKIOgLU, M. 1984 On the formation of ripples on an erodible bed. J. Fluid Mech. 144, 177-190.

UhLMAnN, M. 2005 An immersed boundary method with direct forcing for the simulation of particulate flows. J. Comput. Phys. 209 (2), 448-476.

Venditti, J. G., Church, M. A. \& Bennett, S. J. 2005 Bed form initiation from a flat sand bed. J. Geophys. Res. 110, F01009.

VowincKel, B., KeMPE, T. \& FRÖHLICH, J. 2014 Fluid-particle interaction in turbulent open channel flow with fully-resolved mobile beds. Adv. Water Resour. 72, 32-44. 
Vowinckel, B., Nikora, V. I., KemPe, T. \& FrÖHLICH, J. 2017 Spatially-averaged momentum fluxes and stresses in flows over mobile granular beds: a DNS-based study. J. Hydraul. Res. 55 (2), 208-223.

WONG, Miguel \& PARKeR, GARY. 2006 Reanalysis and correction of bed-load relation of meyer-peter and Müller using their own database. J. Hydraul. Engng ASCE 132 (11), 1159-1168.

WU, J.-S. \& FAETH, G. M. 1993 Sphere wakes in still surroundings at intermediate Reynolds numbers. AIAA J. 31 (8), 1448-1455.

YALIN, M. S. 1977 Mechanics of Sediment Transport, vol. 2. Pergamon Press.

YALIN, M. S. 1985 On the determination of ripple geometry. J. Hydraul. Engng ASCE 111 (8), 1148-1155.

ZENG, L., BALACHANDAR, S. \& NAJJAR, F. M. 2010 Wake response of a stationary finite-sized particle in a turbulent channel flow. Int. J. Multiphase Flow 36, 406-422.

ZGHeIB, N. \& BALACHANDAR, S. 2019 Linear stability analysis of subaqueous bedforms using direct numerical simulations. Theor. Comput. Fluid Dyn. 33 (2), 161-180.

Zgheib, N., Fedele, J. J., Hoyal, D. C. J. D., Perillo, M. M. \& Balachandar, S. 2018 Direct numerical simulation of transverse ripples: 2. Self-similarity, bedform coarsening, and effect of neighboring structures. J. Geophys. Res. 123 (3), 478-500. 